\title{
A checklist of polypores of Kerala state, India
}

\author{
Adarsh $\mathbf{C K}^{1 *}$, Vidyasagran $\mathbf{K}^{1}$ and Ganesh $\mathbf{P N}^{2}$ \\ ${ }^{1}$ Department of Natural Resource Management, College of forestry, Kerala Agricultural University, Kerala, India \\ ${ }^{2}$ Department of Botany, Sree Krishna College, Guruvayoor, Thrissur, Kerala
}

Adarsh CK, Vidyasagran K, Ganesh PN 2018 - A checklist of polypores of Kerala state, India. Studies in Fungi 3(1), 202-226, Doi 10.5943/sif/3/1/21

\begin{abstract}
A literature-based checklist of polypores reported from Kerala State, India is deduced. Altogether 148 species of polypores under 8 families belonging to 68 genera are recorded. The checklist is prepared after reviewing all the available literature and most of them have been published during the last two decades. The geographical distribution of polypores is presented districts wise, including all the 14 districts of Kerala state. The family Polyporaceae has the highest number of species (80), followed by Hymenochaetaceae (37), Fomitopsidaceae (9), Meripilaceae (7), Ganodermataceae (7), Meruliaceae (5), Schizoporaceae (2) and Phanerochaetaceae with a single species. The genus Trametes had the highest representation with 16 species followed by Lentinus and Phellinus with 13 species each. There is no comprehensive studies have been undertaken on the diversity, distribution and functional attributes of polypore fungi in Kerala parts of Western Ghats, which undoubtedly hosting the highest mycodiversity This list is inconclusive and covers only a part of the actual diversity of polypores in Kerala as most of the forest areas are unexplored for the polypore diversity documentation.
\end{abstract}

Key words - Basidiomycota - Diversity - Polyporales - Kerala - Forest

\section{Introduction}

Kerala lies in the south-west corner of peninsular India and is positioned between $8^{\circ} 18^{\prime}$ and $12^{\circ} 48^{\prime}$ north latitudes and $74^{\circ} 52^{\prime}$ and $72^{\circ} 22^{\prime}$ east longitudes (Fig. 1). It is bounded by the Western Ghats mountain ranges to the east and the Arabian Sea to the west. The Western Ghats, which is one of the biodiversity hotspots and World Heritage Site run parallel to the west coast at a distance ranging from 40-80 km. Most of the forested areas of Kerala are parts of the Western Ghats. The total geographical area of Kerala is approximately 38, 863 square kilometers which is only about $1.8 \%$ of the total geographical area of India. Altitude ranges from sea level to 2,600 $\mathrm{m}$ and the terrain falls into three well marked divisions (Leelavathy \& Ganesh 2000):

1. The hilly tracts undulating from Western Ghats in the east and marked by long spurs, extensive ravines and dense forest,

2. The cultivated plains intersected by numerous river, streams and lagoons and

3. The coastal belt with dense coconut plantations and rice fields.

The tropical humid climate of Kerala with intermittent wet and dry periods is closely associated with two principal monsoons, viz. the South-West Monsoon and the North-East 
Monsoon. The rainfall is distributed in these two monsoons and generally extends from early June to middle of December with comparatively less rainy intervals in September and October. The South-West Monsoon first hits Kerala coast during June. June, July and August are the wettest months. The North-East Monsoon, also called as retreating monsoon, brings less rain in Kerala in the months of October and December. Annual rain fall is approximately 3,000 mm. It is obvious from the above account that Kerala must be harboring a luxuriant mycodiversity (Mohanan 2011). However, there is in no comprehensive studies were conducted for many groups of fungi especially polypores. There are only a few studies regarding the polypore phenology, host specificity, role of substrate features, decay class, forest type and diversity of polypores in tropical forests compared to the temperate forests. Dry weather starts from middle of December and extend up to early June. Highest relative humidity varies from $100 \%$ in June to $80 \%$ in December and lowest from $78 \%$ in July-August to $48 \%$ in January. Temperature generally varies from a monthly mean maximum of $33.6^{\circ} \mathrm{C}$ in April to $27.7^{\circ} \mathrm{C}$ in July and a monthly mean minimum of $26.5^{\circ} \mathrm{C}$ in April to $21^{\circ} \mathrm{C}$ in July (Indian Meteorological Department, Govt. of India).

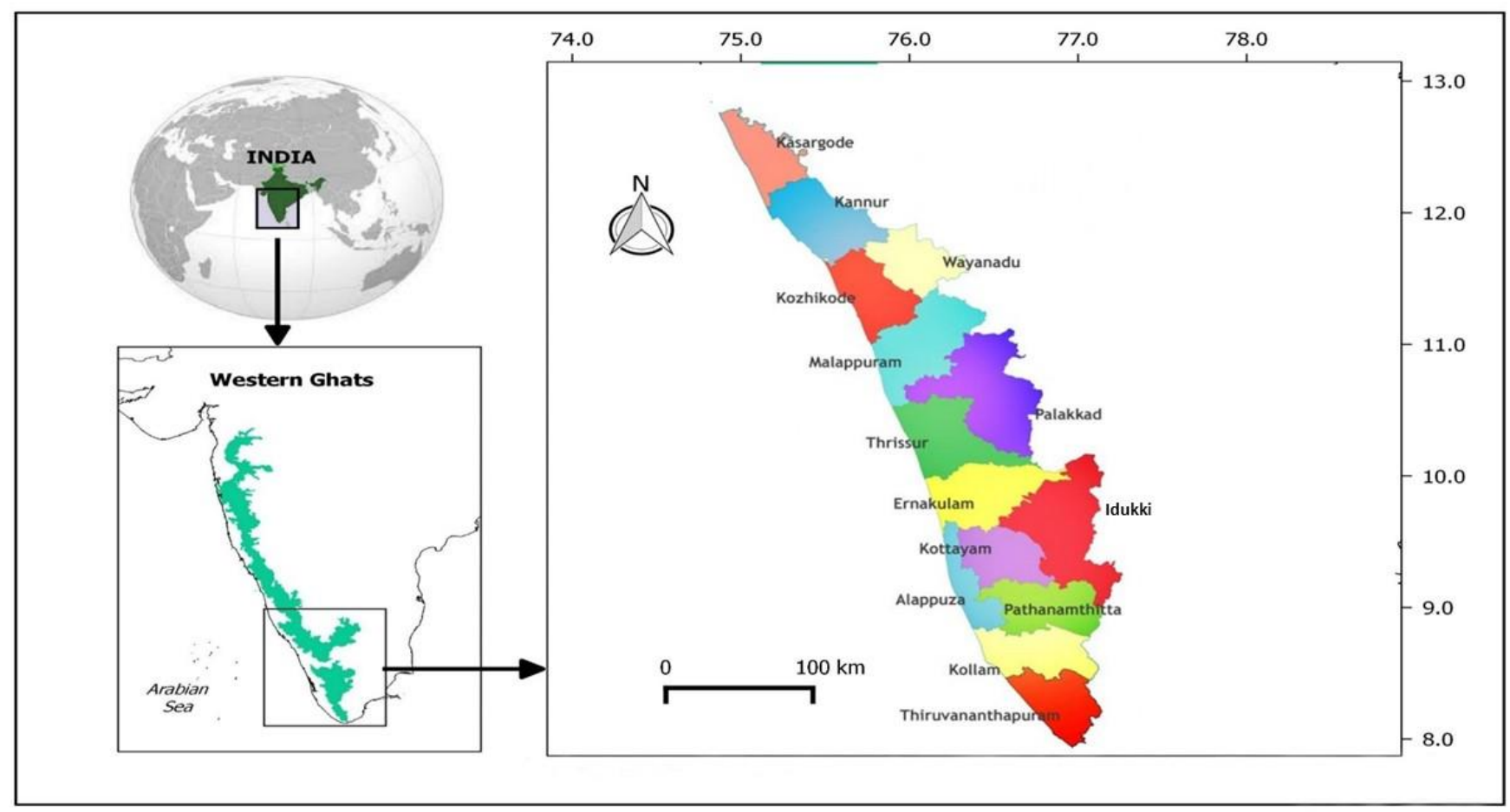

Fig. 1 - Map of Kerala State, India.

The vegetation of Kerala is unusually rich and diverse, almost unique for so small a region. India is divided in to several phytogeographic regions based on static features of geography and physiography. Kerala is in Malabar region which extends along the western side of peninsula from southern Gujarat to Kanyakumari (Leelavathy \& Ganesh 2000). Due to different geological history and relative isolation, the flora of the west coast differs considerably from other region of India. Bordering the eastern side is the Deccan plateau which is considered as the "rain shadow region" of Western Ghats. Major parts of the other southern Indian states like Tamil Nadu, Andhra Pradesh and Karnataka belongs to Deccan plateau.

The abundance of species of Malayan-type, especially plants of Sterculiaceae, Teliaceae, Anacardaceae, Meliaceae, Myrtaceae, Melastomaceae, Gesneriaceae, Piperaceae, Orchidaceae, and Araceae, is one of the most distinctive characteristics of Malabar flora. The presence of taxa belonging to family Clusiaceae, Dipterocarpaceae, Myristicaceae, and many species of Arecaceae 
and Poaceae is also distinctive. Another noteworthy feature is the endemism of some species such as Gluta travancuorica Bedd., Dipterocarpus bourdillonii Brand., and the monotypic genus Blepharistemma Wall. ex Benth. which are found only in Kerala, though allied species are present in the Andamans, Burma and Indo-Malayan regions (Sasidharan 2004).

Among the 4,667 vascular plant species of the State (Sasidharan 2004), 344 are endemic (Reddy et al. 2007). Different vegetational types can be met with in Kerala depending on altitude and consequent variations of rainfall and temperature. In a cross section of the state three main vegetational types can be seen (Leelavathy \& Ganesh 2000):

1. Deciduous forests or monsoon forests from sea level to about $610 \mathrm{~m}$, sometimes rising up to $945 \mathrm{~m}$.

2. Tropical evergreen forests or rain forests between $762 \mathrm{~m}$ and $1067 \mathrm{~m}$, sometimes starting from $610 \mathrm{~m}$ itself and merging in to monsoon forests.

3. Temperate evergreeen forest formations at an elevation above $1524 \mathrm{~m}$, with all conceivable grades between the main zones.

The very high biological diversity of Kerala is primarily due to the highly diversified ecological niches encompassing evergreen forests, semi-evergreen forests, deciduous forests shola forests, grasslands, mangroves, dry teak forests, lateritic semi-evergreen forests, littoral forests, myristica swamps, Nilgiri subtropical hill forests, south Indian subtropical hill savannah, southern moist mixed deciduous forests, southern secondary moist mixed deciduous forests and very moist teak forests (Farook et al. 2013).

Most of the natural forests are fast degrading due to deforestation and other human interference and true virgin forests are almost lacking. No comprehensive studies have been undertaken on the diversity, distribution and functional attributes of polypore fungi in Kerala parts of Western Ghats, which undoubtedly is also hosting the highest mycodiversity. There are several reasons for this scenario, the scarcity of trained mycologists in the State being a major one. In order to consolidate the information so far made available on the diversity of polypores of this region and to contribute to the knowledge of mycota of Kerala, here a literature-based checklist of polypores reported from Kerala State.

\section{Materials \& Methods}

This checklist is prepared from the information collected only from the publications up to April 2018 excluding all unpublished Masters, M.Phil and Ph.D dissertations. Species which is identified only up to genus level is excluded in this checklist. This checklist provides no herbaria data as that information is available in the references given. The systematic arrangement and the nomenclature used here are according to Index Fungorum (www.indexfungorum.org) as accessed on 20th April 2018. In this checklist, the names of some species as reported in the cited publications have been given and the currently accepted name according to the Index Fungorum (www.indexfungorum.org), has also added (Bold). Invalidly proposed new species from the region are excluded from this list. The checklist is organized alphabetically by order, family, genus and species

\section{Results}

148 species of polypores belong to 68 genera were reported from Kerala State on the basis of published information (Table 1). Most of these papers were published during last two decades. The geographical distribution of the reports covers all the 14 districts of the State (Fig. 1). Although the list in Table 1 might appear extensive and contains 148 species, it covers only a part of the actual diversity of polypores in Kerala. Most surveys have been conducted in a few, accessible areas of the State. The explorations on polypore diversity in most of the dense forests of the State is still insuffcient. The studies on the polypores of the state have been incomplete, inadequate and therefore inconclusive. 
Table 1 Species of polypores reported from Kerala State, India

\begin{tabular}{|c|c|c|c|c|}
\hline & Scientific Name & \multicolumn{2}{|c|}{ DISTRIBUTION } & \multirow[t]{2}{*}{ References } \\
\hline & & District & Locations & \\
\hline (I) & \multicolumn{4}{|c|}{ FAMILY : FOMITOPSIDACEAE } \\
\hline 1. & $\begin{array}{l}\text { Antrodia albida (Fr.) } \\
\text { Donk } 1966\end{array}$ & Wayanad & Chandanathode & Mohanan 2011 \\
\hline \multirow[t]{9}{*}{2.} & \multirow{3}{*}{$\begin{array}{l}\text { Daedalea dochmia (Berk. } \\
\text { \& Broome) T. Hatt. } 2005\end{array}$} & Wayanad & Chembra peak & Mohanan 2011 \\
\hline & & Wayanad & Kuruva, Begur & Mohanan 2011 \\
\hline & & Ernakulam & $\begin{array}{l}\text { Iringole kavu, } \\
\text { Perumbavoor }\end{array}$ & Mohanan 2011 \\
\hline & \multirow{6}{*}{$\begin{array}{l}\text { Fomitopsis dochmia } \\
\text { (Berk. \& Broome) } \\
\text { Ryvarden } 1972\end{array}$} & Idukki & Mankulam & Mohanan 2011 \\
\hline & & Thrissur & Peechi & $\begin{array}{l}\text { Florence \& } \\
\text { Yesodharan } 2000\end{array}$ \\
\hline & & Thrissur & $\begin{array}{l}\text { Perumpara (W), } \\
\text { Malakkappara, Sholayar }\end{array}$ & $\begin{array}{l}\text { Leelavathy \& } \\
\text { Ganesh } 2000\end{array}$ \\
\hline & & Wayanad & $\begin{array}{l}\text { Kanoth forest Reserve, } \\
\text { Chundanthody }\end{array}$ & $\begin{array}{l}\text { Leelavathy \& } \\
\text { Ganesh } 2000\end{array}$ \\
\hline & & Palakkad & $\begin{array}{l}\text { Panthemthode, Silent } \\
\text { Valley NP, } \\
\text { Parambikulam } \\
\end{array}$ & $\begin{array}{l}\text { Leelavathy \& } \\
\text { Ganesh } 2000\end{array}$ \\
\hline & & Thiruvananthapuram & Ponmudi & Mohanan 2011 \\
\hline \multirow[t]{2}{*}{3.} & \multirow{2}{*}{$\begin{array}{l}\text { Hexagonia sulcata Berk. } \\
1847 \\
\\
\text { Daedalea sulcata (Berk.) } \\
\text { Ryvarden } 1977\end{array}$} & Palakkad & $\begin{array}{l}\text { Panthamthode \& } \\
\text { Poochippara, Silent } \\
\text { Valley NP }\end{array}$ & $\begin{array}{l}\text { Leelavathy \& } \\
\text { Ganesh } 2000\end{array}$ \\
\hline & & Palakkad & Silent Valley NP & Mohanan 2011 \\
\hline \multirow[t]{5}{*}{4.} & \multirow{5}{*}{$\begin{array}{l}\text { Fomitopsis palustris } \\
\text { (Berk. \& M.A. Curtis) } \\
\text { Gilb. \& Ryvarden } 1985\end{array}$} & Pathanamthitta & \begin{tabular}{|l|} 
Chalakkayam \\
\end{tabular} & Mohanan 1994 \\
\hline & & Palakkad & Silent Valley NP & Mohanan 1994 \\
\hline & & Wayanad & Kottiyur & Mohanan 1994 \\
\hline & & Malappuram & $\begin{array}{l}\text { Calicut University } \\
\text { Campus }\end{array}$ & $\begin{array}{l}\text { Leelavathy \& } \\
\text { Ganesh } 2000\end{array}$ \\
\hline & & Thrissur & Patturaical & $\begin{array}{l}\text { Leelavathy \& } \\
\text { Ganesh } 2000\end{array}$ \\
\hline 5. & $\begin{array}{l}\text { Polyporus ostreiformis } \\
\text { Berk. } 1878 \\
\text { Fomitopsis ostreiformis } \\
\text { (Berk.) T. Hatt. } 2003 \\
\end{array}$ & Thrissur & Peechi & $\begin{array}{l}\text { Florence \& } \\
\text { Yesodharan } 2000\end{array}$ \\
\hline \multirow[t]{2}{*}{6.} & \multirow{2}{*}{$\begin{array}{l}\text { Laetiporus sulphureus } \\
\text { (Bull.) Murrill } 1920\end{array}$} & Idukki & Mannavanshola, Munnar & Mohanan 2011 \\
\hline & & Ernakulam & $\begin{array}{l}\text { Iringole kavu, } \\
\text { Perumbavoor }\end{array}$ & Mohanan 2011 \\
\hline 7. & $\begin{array}{l}\text { Antrodia serialis (Fr.) } \\
\text { Donk } 1966 \\
\text { Neoantrodia serialis (Fr.) } \\
\text { Audet } 2017\end{array}$ & Ernakulam & $\begin{array}{l}\text { Iringole kavu, } \\
\text { Perumbavoor }\end{array}$ & Mohanan 2011 \\
\hline \multirow[t]{2}{*}{8.} & \multirow{2}{*}{$\begin{array}{l}\text { Tyromyces pelliculosus } \\
\text { (Berk.) G. Cunn. } 1965 \\
\text { Postia pelliculosa (Berk.) } \\
\text { Rajchenb. } 1988 \\
\text { Oligoporus pelliculosus } \\
\text { (Berk.) Ryvarden } 2018\end{array}$} & Wayanad & Kuppadi, Sultan Battery & $\begin{array}{l}\text { Leelavathy \& } \\
\text { Ganesh } 2000\end{array}$ \\
\hline & & Wayanad & Chethalayam & Mohanan 2011 \\
\hline 9. & $\begin{array}{l}\text { Rhodofomitopsis feei (Fr.) } \\
\text { B.K. Cui, M.L. Han \& } \\
\text { Y.C. Dai } 2016\end{array}$ & Malappuram & $\begin{array}{l}\text { Calicut University } \\
\text { Campus, } \\
\text { Kanjiramkadavu, } \\
\text { Amarambalam, } \\
\text { Nilambur } \\
\end{array}$ & $\begin{array}{l}\text { Leelavathy \& } \\
\text { Ganesh } 2000\end{array}$ \\
\hline
\end{tabular}


Table 1 Continued.

\begin{tabular}{|c|c|c|c|c|}
\hline & Scientific Name & \multicolumn{2}{|c|}{ DISTRIBUTION } & \multirow[t]{2}{*}{ References } \\
\hline & & District & Locations & \\
\hline (I) & \multicolumn{4}{|c|}{ FAMILY : FOMITOPSIDACEAE } \\
\hline & & Palakkad & $\begin{array}{l}\text { Paanthamthode, Silent } \\
\text { Valley NP }\end{array}$ & $\begin{array}{l}\text { Leelavathy \& } \\
\text { Ganesh } 2000\end{array}$ \\
\hline & & Idukki & $\begin{array}{l}\text { Pampadumshola, } \\
\text { Munnar }\end{array}$ & Mohanan 2011 \\
\hline & & Pathanamthitta & Mannarapara, Konni & Mohanan 2011 \\
\hline (II) & \multicolumn{4}{|c|}{ FAMILY: GANODERMATACEAE } \\
\hline \multirow[t]{3}{*}{10.} & \multirow{3}{*}{$\begin{array}{l}\text { Amauroderma } \\
\text { conjunctum }(\text { Lloyd) } \\
\text { Torrend } 1920\end{array}$} & Thrissur & Sidhan pocket,Sholayar & Mohanan 2011 \\
\hline & & Thiruvananthapuram & Sasthanada, Arippa & Mohanan 2011 \\
\hline & & Thiruvananthapuram & $\begin{array}{l}\text { Ammayambalam, } \\
\text { Arippa }\end{array}$ & Mohanan 2011 \\
\hline \multirow[t]{2}{*}{11.} & \multirow[t]{2}{*}{$\begin{array}{l}\text { Amauroderma fuscoporia } \\
\text { Wakef. } 1948\end{array}$} & Ernakulam & $\begin{array}{l}\text { Iringole kavu, } \\
\text { Perumbavoor }\end{array}$ & Mohanan 2011 \\
\hline & & Pallakkad & Nelliyampathy & Mohanan 2011 \\
\hline \multirow[t]{5}{*}{12.} & \multirow{5}{*}{$\begin{array}{l}\text { Amauroderma rugosum } \\
\text { (Blume \& T. Nees) } \\
\text { Torrend } 1920\end{array}$} & Thrissur & Vazhachhal & $\begin{array}{l}\text { Leelavathy \& } \\
\text { Ganesh } 2000\end{array}$ \\
\hline & & Malappuram & $\begin{array}{ll}\begin{array}{l}\text { Calicut } \\
\text { campus }\end{array} & \text { university } \\
\end{array}$ & $\begin{array}{l}\text { Leelavathy \& } \\
\text { Ganesh } 2000\end{array}$ \\
\hline & & Kannur & Pazhayangadi & $\begin{array}{l}\text { Leelavathy \& } \\
\text { Ganesh } 2000\end{array}$ \\
\hline & & Thrissur & Peechi & $\begin{array}{l}\text { Florence \& } \\
\text { Yesodharan } 2000\end{array}$ \\
\hline & & Pathanamthitta & Thenmala & Mohanan 2011 \\
\hline \multirow[t]{9}{*}{13.} & \multirow{9}{*}{$\begin{array}{l}\text { Ganoderma applanatum } \\
\text { (Pers.) Pat. } 1887\end{array}$} & Wayanad & Chethalayam & Mohanan 2011 \\
\hline & & Wayanad & Muthanga & Mohanan 2011 \\
\hline & & Idukki & Vattavada, Munnar & Mohanan 2011 \\
\hline & & Idukki & $\begin{array}{l}\text { Pampadumshola, } \\
\text { Munnar }\end{array}$ & Mohanan 2011 \\
\hline & & Ernakulam & $\begin{array}{l}\text { Iringole kavu, } \\
\text { Perumbavoor }\end{array}$ & Mohanan 2011 \\
\hline & & Idukki & Anamudishola, Munnar & Mohanan 2011 \\
\hline & & Wayanad & Periya & Mohanan 2011 \\
\hline & & Thrissur & Peechi & Mohanan 2011 \\
\hline & & Malappuram & Nadugani, Nilambur & \\
\hline \multirow[t]{5}{*}{14.} & \multirow[t]{5}{*}{$\begin{array}{l}\text { Ganoderma australe (Fr.) } \\
\text { Pat. } 1889\end{array}$} & Palakkad & $\begin{array}{l}\text { Parambikulam, } \\
\text { Chattappuram }\end{array}$ & $\begin{array}{l}\text { Leelavathy \& } \\
\text { Ganesh } 2000\end{array}$ \\
\hline & & Malappuram & $\begin{array}{l}\text { Calicut university } \\
\text { campus }\end{array}$ & $\begin{array}{l}\text { Leelavathy \& } \\
\text { Ganesh } 2000\end{array}$ \\
\hline & & Wayanad & $\begin{array}{l}\text { Muthanga, Mundakaith, } \\
\text { Kanoth reserve forest, }\end{array}$ & $\begin{array}{l}\text { Leelavathy \& } \\
\text { Ganesh } 2000\end{array}$ \\
\hline & & Thrissur & Malakkappara & $\begin{array}{l}\text { Leelavathy \& } \\
\text { Ganesh } 2000\end{array}$ \\
\hline & & Calicut & Velangad & $\begin{array}{l}\text { Leelavathy \& } \\
\text { Ganesh } 2000\end{array}$ \\
\hline \multirow[t]{3}{*}{15.} & \multirow{3}{*}{$\begin{array}{l}\text { Ganoderma colossus (Fr.) } \\
\text { C.F. Baker } 1920\end{array}$} & Thiruvananthapuram & Arippa & Mohanan 2011 \\
\hline & & Malappuram & Nilambur & Mohanan 2011 \\
\hline & & Palakkad & Nelliyampathy & Mohanan 2011 \\
\hline \multirow[t]{3}{*}{16.} & \multirow[t]{3}{*}{$\begin{array}{l}\text { Ganoderma lucidum } \\
\text { (Curtis) P. Karst. } 1881\end{array}$} & Malappuram & $\begin{array}{l}\text { Calicut university } \\
\text { campus }\end{array}$ & $\begin{array}{l}\text { Leelavathy \& } \\
\text { Ganesh } 2000\end{array}$ \\
\hline & & Thrissur & $\begin{array}{l}\text { Sholayar, Kannara, } \\
\text { Peechi }\end{array}$ & $\begin{array}{l}\text { Leelavathy \& } \\
\text { Ganesh } 2000\end{array}$ \\
\hline & & Malappuram & Nedumgayam-Nilambur & $\begin{array}{l}\text { Leelavathy \& } \\
\text { Ganesh } 2000\end{array}$ \\
\hline
\end{tabular}


Table 1 Continued.

\begin{tabular}{|c|c|c|c|c|}
\hline & Scientific Name & \multicolumn{2}{|l|}{ DISTRIBUTION } & \multirow[t]{2}{*}{ References } \\
\hline & & District & Locations & \\
\hline (II) & \multicolumn{4}{|c|}{ FAMILY: GANODERMATACEAE } \\
\hline & & Wayanad & $\begin{array}{l}\text { Meppadi, Kanoth reserve } \\
\text { forest }\end{array}$ & $\begin{array}{l}\text { Leelavathy \& } \\
\text { Ganesh } 2000\end{array}$ \\
\hline & & Idukki & Munnar & Mohanan 2011 \\
\hline & & Wayanad & Kuruva, Begur & Mohanan 2011 \\
\hline & & Idukki & Devikulam, Munnar & Mohanan 2011 \\
\hline & & Idukki & Vattavada, Munnar & Mohanan 2011 \\
\hline & & Idukki & Mannavanshola, Munnar & Mohanan 2011 \\
\hline (III) & \multicolumn{4}{|c|}{ FAMILY : HYMENOCHAETACEAE } \\
\hline 17. & $\begin{array}{l}\text { Coltricia cinnamomea } \\
\text { (Jacq.) Murrill } 1904\end{array}$ & Ernakulam & $\begin{array}{l}\text { Iringole kavu, } \\
\text { Perumbavoor }\end{array}$ & Mohanan 2011 \\
\hline 18. & $\begin{array}{l}\text { Coltricia focicola (Berk. } \\
\text { \& M.A. Curtis) Murrill } \\
1908\end{array}$ & Malappuram & $\begin{array}{l}\text { Calicut University } \\
\text { Campus }\end{array}$ & $\begin{array}{l}\text { Leelavathy \& } \\
\text { Ganesh } 2000\end{array}$ \\
\hline \multirow[t]{3}{*}{19.} & \multirow[t]{3}{*}{$\begin{array}{l}\text { Cyclomyces setiporus } \\
\text { (Berk.) Pat. } 1900\end{array}$} & Malappuram & $\begin{array}{l}\text { Chanddhakkunnu, } \\
\text { Nilambur }\end{array}$ & Mohanan 2011 \\
\hline & & Idukkki & Anamudishola & \\
\hline & & Malappuram & Nadukani (Nilambur) & $\begin{array}{l}\text { Leelavathy \& } \\
\text { Ganesh } 2000\end{array}$ \\
\hline \multirow[t]{3}{*}{20.} & \multirow[t]{3}{*}{$\begin{array}{l}\text { Cyclomyces tabacinus } \\
\text { (Mont.) Pat. } 1900\end{array}$} & Kasaragod & $\begin{array}{l}\text { Kerala Kanataka state } \\
\text { boundary }\end{array}$ & $\begin{array}{l}\text { Leelavathy \& } \\
\text { Ganesh } 2000\end{array}$ \\
\hline & & Malappuram & $\begin{array}{l}\text { Kaduvakunnu } \\
\text { (Nadukani-Nilambur) }\end{array}$ & $\begin{array}{l}\text { Leelavathy \& } \\
\text { Ganesh } 2000\end{array}$ \\
\hline & & Wayanad & Kathiyur forest Reserve & $\begin{array}{l}\text { Leelavathy \& } \\
\text { Ganesh } 2000\end{array}$ \\
\hline \multirow[t]{3}{*}{21.} & \multirow{3}{*}{$\begin{array}{l}\text { Phellinus contiguus (Pers.) } \\
\text { Pat. } 1928\end{array}$} & Wayanad & Kottiyoor & Mohanan 1994 \\
\hline & & Malappuram & Cherupuzha, Nilambur & $\begin{array}{l}\text { Leelavathy \& } \\
\text { Ganesh } 2000\end{array}$ \\
\hline & & Pathanamthitta & Aramba, Achenkoil & $\begin{array}{l}\text { Leelavathy \& } \\
\text { Ganesh } 2000\end{array}$ \\
\hline 22. & $\begin{array}{l}\text { Phellinus caryophylleus } \\
\text { (Cooke) Ryvarden } 1972\end{array}$ & Thrissur & $\begin{array}{l}\text { Nelliyampadam, } \\
\text { Thrumani }\end{array}$ & $\begin{array}{l}\text { Florence \& } \\
\text { Yesodharan } 2000\end{array}$ \\
\hline \multirow[t]{2}{*}{23.} & \multirow{2}{*}{$\begin{array}{l}\text { Fomes setulosus Lloyd } \\
1915 \\
\text { Phellinus setulosus } \\
\text { (Lloyd) Imazeki, (1943) }\end{array}$} & Wayanad & - & $\begin{array}{l}\text { Bagchee 1954, } \\
\text { Rangaswami et al. } \\
\text { 1970, Bilgrami et } \\
\text { al. 1991 }\end{array}$ \\
\hline & & Thrissur & Peechi & $\begin{array}{l}\text { Florence \& } \\
\text { Yesodharan 2000, } \\
\text { Florence 2004 }\end{array}$ \\
\hline \multirow[t]{2}{*}{24.} & \multirow{2}{*}{\begin{tabular}{|l|} 
Phellinus punctatus (P. \\
Karst.) Pilát 1942 \\
\\
Fomitiporia punctata (P. \\
Karst.) Murrill 1947 \\
\end{tabular}} & Malappuram & $\begin{array}{l}\text { Calicut University } \\
\text { Campus }\end{array}$ & $\begin{array}{l}\text { Ganesh \& } \\
\text { Leelavathy } 1986 \\
\text { Leelavathy \& } \\
\text { Ganesh } 2000 \\
\end{array}$ \\
\hline & & Idukki & Mannavanshola, Munnar & Mohanan 2011 \\
\hline 25. & $\begin{array}{l}\text { Phellinus gilvoides (Petch) } \\
\text { Ryvarden } 1972 \\
\text { Fulvifomes cesatii (Bres.) } \\
\text { Y.C. Dai 2010 }\end{array}$ & Wayanad & Periya Reserve Forest & $\begin{array}{l}\text { Leelavathy \& } \\
\text { Ganesh } 2000\end{array}$ \\
\hline 26. & $\begin{array}{l}\text { Phellinus durissimus } \\
\text { (Lloyd) A. Roy } 1979\end{array}$ & Thiruvananthapuram & Kallar, Ponmudi & Mohanan 2011 \\
\hline
\end{tabular}


Table 1 Continued.

\begin{tabular}{|c|c|c|c|c|}
\hline & Scientific Name & \multicolumn{2}{|c|}{ DISTRIBUTION } & \multirow[t]{2}{*}{ References } \\
\hline & & District & Locations & \\
\hline (III) & \multicolumn{4}{|c|}{ FAMILY : HYMENOCHAETACEAE } \\
\hline & $\begin{array}{l}\text { Fulvifomes durissimus } \\
\text { (Lloyd) Bondartseva \& S. } \\
\text { Herrera } 1992\end{array}$ & & & \\
\hline 27. & $\begin{array}{l}\text { Aurificaria indica } \\
\text { (Massee) D.A. Reid } 1963 \\
\\
\text { Fulvifomes indicus } \\
\text { (Massee) L.W. Zhou } \\
\text { 2014 }\end{array}$ & Wayanad & Manikunumala & Mohanan 2011 \\
\hline 28. & $\begin{array}{l}\text { Phellinus rhytiphloeus } \\
\text { (Mont.) Ryvarden } 1980 \\
\\
\text { Fulvifomes rhytiphloeus } \\
\text { (Mont.) Camp.-Sant. \& } \\
\text { Robledo 2015 }\end{array}$ & Palakkad & Nelliyampathy & Mohanan 2011 \\
\hline \multirow[t]{5}{*}{29.} & \multirow[t]{2}{*}{$\begin{array}{l}\text { Phellinus ferreus (Pers.) } \\
\text { Bourdot \& Galzin } 1928\end{array}$} & Ernakulam & Malayattoor & $\begin{array}{l}\text { Sankaran \& } \\
\text { Florence } 1995\end{array}$ \\
\hline & & Thrissur & Peechi WLS & $\begin{array}{l}\text { Florence \& } \\
\text { Yesodharan 1997, } \\
2000\end{array}$ \\
\hline & \multirow[t]{3}{*}{$\begin{array}{l}\text { Fuscoporia ferrea (Pers.) } \\
\text { G. Cunn. } 1948\end{array}$} & Pathanamthitta & Aramba, Achankoil & $\begin{array}{l}\text { Leelavathy \& } \\
\text { Ganesh } 2000\end{array}$ \\
\hline & & Wayanad & Periya & Mohanan 2011 \\
\hline & & Idukki & $\begin{array}{l}\text { Pampadumshola, } \\
\text { Munnar }\end{array}$ & Mohanan 2011 \\
\hline \multirow[t]{2}{*}{30.} & $\begin{array}{l}\text { Phellinus ferruginosus } \\
\text { (Schrad.) Pat. } 1900\end{array}$ & Ernakulam & $\begin{array}{l}\text { Iringole kavu, } \\
\text { Perumbavoor }\end{array}$ & Mohanan 2011 \\
\hline & $\begin{array}{l}\text { Fuscoporia ferruginosa } \\
\text { (Schrad.) Murrill (1907) }\end{array}$ & & & \\
\hline \multirow[t]{5}{*}{31.} & \multirow{5}{*}{$\begin{array}{l}\text { Phellinus senex (Nees \& } \\
\text { Mont.) Imazeki } 1952\end{array}$} & Palakkad & Nelliyampathy, & Mohanan 2011 \\
\hline & & Wayanad & Muthanga & \\
\hline & & Pathanamthitta & Konni & \\
\hline & & Malappuram & $\begin{array}{l}\text { Calicut University } \\
\text { Campus }\end{array}$ & $\begin{array}{l}\text { Leelavathy \& } \\
\text { Ganesh } 2000\end{array}$ \\
\hline & & Thrissur & Vazhachal & $\begin{array}{l}\text { Leelavathy \& } \\
\text { Ganesh } 2000\end{array}$ \\
\hline \multirow[t]{2}{*}{32.} & \multirow{2}{*}{$\begin{array}{l}\text { Phellinus wahlbergii (Fr.) } \\
\text { D.A. Reid } 1975 \\
\\
\text { Fuscoporia wahlbergii } \\
\text { (Fr.) T. Wagner \& M. } \\
\text { Fisch. 2001 }\end{array}$} & Palakkad & $\begin{array}{l}\text { Walakkad, Silent Valley } \\
\text { NP }\end{array}$ & $\begin{array}{l}\text { Leelavathy \& } \\
\text { Ganesh } 2000\end{array}$ \\
\hline & & Idukki & Mannavanshola, Munnar & Mohanan 2011 \\
\hline \multirow[t]{3}{*}{33.} & \multirow{3}{*}{$\begin{array}{l}\text { Hymenochaete rubiginosa } \\
\text { (Dicks.) Lév. } 1846\end{array}$} & Malappuram & Nilambur & Mohanan 2011 \\
\hline & & Ernakulam & $\begin{array}{l}\text { Iringole kavu, } \\
\text { Perumbavoor }\end{array}$ & \\
\hline & & Idukki & $\begin{array}{l}\text { Mannavanshola, } \\
\text { Idukki }\end{array}$ & \\
\hline 34. & $\begin{array}{l}\text { Pseudochaete tabacina } \\
\text { (Sowerby) T. Wagner \& } \\
\text { M. Fisch. } 2002\end{array}$ & Wayanad & Chembra peak & Mohanan 2011 \\
\hline
\end{tabular}


Table 1 Continued.

\begin{tabular}{|c|c|c|c|c|}
\hline & Scientific Name & \multicolumn{2}{|c|}{ DISTRIBUTION } & \multirow[t]{2}{*}{ References } \\
\hline & & District & Locations & \\
\hline (III) & \multicolumn{4}{|c|}{ FAMILY : HYMENOCHAETACEAE } \\
\hline & $\begin{array}{l}\text { Hymenochaetopsis } \\
\text { tabacina (Sowerby) S.H. } \\
\text { He \& Jiao Yang } 2016\end{array}$ & & & \\
\hline 35. & $\begin{array}{l}\text { Phellinus sublinteus } \\
\text { (Murrill) Ryvarden } 1972 \\
\text { Inonotus luteoumbrinus } \\
\text { (Romell) Ryvarden } 2005\end{array}$ & Malappuram & $\begin{array}{l}\text { Calicut University } \\
\text { Campus }\end{array}$ & $\begin{array}{l}\text { Leelavathy \& } \\
\text { Ganesh } 2000\end{array}$ \\
\hline 36. & $\begin{array}{l}\text { Inonotus nothofagi G. } \\
\text { Cunn. } 1948\end{array}$ & Idukki & Chinar & Mohanan 2011 \\
\hline \multirow[t]{2}{*}{37.} & \multirow{2}{*}{$\begin{array}{l}\text { Phellinus pachyphloeus } \\
\text { (Pat.) Pat. } 1900 \\
\\
\text { Inonotus pachyphloeus } \\
\text { (Pat.) T. Wagner \& M. } \\
\text { Fisch. 2002 }\end{array}$} & Ernakulam & $\begin{array}{l}\text { Iringole kavu, } \\
\text { Perumbavoor }\end{array}$ & Mohanan 2011 \\
\hline & & Wayanad & Kuruva, Begur & Mohanan 2011 \\
\hline 38. & $\begin{array}{l}\text { Inonotus patouillardii } \\
\text { (Rick) Imazeki } 1943\end{array}$ & Malappuram & $\begin{array}{l}\text { Chandhakunnu, } \\
\text { Nilambur }\end{array}$ & Mohanan 2011 \\
\hline \multirow[t]{4}{*}{39.} & \multirow{4}{*}{$\begin{array}{l}\text { Innotus tabacinus } \\
\text { (Mont.) G. Cunn. } 1948\end{array}$} & Wayanad & Chandanathode & Mohanan 2011 \\
\hline & & Thrissur & $\begin{array}{l}\text { Pukayilappara, } \\
\text { Vazhachal }\end{array}$ & Mohanan 2011 \\
\hline & & Idukki & Mannavanshola & Mohanan 2011 \\
\hline & & Thiruvananthapuram & Kallar, Ponmudi & Mohanan 2011 \\
\hline \multirow[t]{2}{*}{40.} & \multirow{2}{*}{$\begin{array}{l}\text { Phellinus conchatus } \\
\text { (Pers.) Quél. } 1886 \\
\\
\text { Phellinopsis conchata } \\
\text { (Pers.) Y.C. Dai } 2010\end{array}$} & Malappuram & $\begin{array}{l}\text { Cherupuzha river } \\
\text { (Nilambur) }\end{array}$ & $\begin{array}{l}\text { Leelavathy \& } \\
\text { Ganesh } 2000\end{array}$ \\
\hline & & Pathanamthitta & Aramba (Achankoil) & $\begin{array}{l}\text { Leelavathy \& } \\
\text { Ganesh } 2000\end{array}$ \\
\hline \multirow[t]{2}{*}{41.} & \multirow[t]{2}{*}{$\begin{array}{l}\text { Phellinus adamantinus } \\
\text { (Berk.) Ryvarden } 1972\end{array}$} & Malappuram & $\begin{array}{l}\begin{array}{l}\text { Kaduvakunnu } \\
\text { (Nadukani-Nilambur) }\end{array} \\
\end{array}$ & $\begin{array}{l}\text { Leelavathy \& } \\
\text { Ganesh } 2000\end{array}$ \\
\hline & & Wayanad & Kuruva, Begur & Mohanan 2011 \\
\hline \multirow[t]{2}{*}{42.} & \multirow[t]{2}{*}{$\begin{array}{l}\text { Phellinus allardii (Bres.) } \\
\text { S. Ahmad } 1972\end{array}$} & Palakkad & $\begin{array}{l}\text { Thunacadav } \\
\text { (Parambikulam tiger } \\
\text { reserve) }\end{array}$ & $\begin{array}{l}\text { Leelavathy \& } \\
\text { Ganesh } 2000\end{array}$ \\
\hline & & Wayanad & Kottiyoor & Mohanan 1994 \\
\hline \multirow[t]{4}{*}{43.} & \multirow[t]{4}{*}{$\begin{array}{l}\text { Phellinus fastuosus (Lév.) } \\
\text { S. Ahmad } 1972\end{array}$} & Ernakulam & Malayattoor & $\begin{array}{l}\text { Sankaran \& } \\
\text { Florence } 1995\end{array}$ \\
\hline & & Thrissur & Peechi WLS & $\begin{array}{l}\text { Florence \& } \\
\text { Yesodharan 1997, } \\
2000\end{array}$ \\
\hline & & Malappuram & Nilambur, Karulai & $\begin{array}{l}\text { Leelavathy \& } \\
\text { Ganesh 2000, } \\
\text { Mohanan 2011 } \\
\end{array}$ \\
\hline & & Palakkad & $\begin{array}{l}\text { Thunakadavu } \\
\text { (Parambikulam Tiger } \\
\text { Reserve) }\end{array}$ & $\begin{array}{l}\text { Leelavathy \& } \\
\text { Ganesh } 2000\end{array}$ \\
\hline 44. & $\begin{array}{l}\text { Phellinus } \\
\text { ferrugineovelutinus } \\
\text { (Henn.) Ryvarden } 1972\end{array}$ & Wayanad & Meppadi & $\begin{array}{l}\text { Leelavathy \& } \\
\text { Ganesh } 2000\end{array}$ \\
\hline
\end{tabular}


Table 1 Continued.

\begin{tabular}{|c|c|c|c|c|}
\hline & Scientific Name & \multicolumn{2}{|c|}{ DISTRIBUTION } & \multirow[t]{2}{*}{ References } \\
\hline & & District & Locations & \\
\hline (III) & \multicolumn{4}{|c|}{ FAMILY : HYMENOCHAETACEAE } \\
\hline \multirow[t]{10}{*}{45.} & \multirow[t]{10}{*}{\begin{tabular}{|l|} 
Phellinus gilvus \\
(Schwein.) Pat. 1900
\end{tabular}} & Ernakulam & Malayattoor & $\begin{array}{l}\text { Sankaran \& } \\
\text { Florence } 1995\end{array}$ \\
\hline & & Thrissur & Peechi WLS & $\begin{array}{l}\text { Florence \& } \\
\text { Yesodharan 1997, } \\
2000\end{array}$ \\
\hline & & Wayanad & $\begin{array}{l}\text { Kuppadi, Periya Reserve } \\
\text { Forest, } \\
\text { Meppadi }\end{array}$ & $\begin{array}{l}\text { Leelavathy \& } \\
\text { Ganesh } 2000\end{array}$ \\
\hline & & Palakkad & $\begin{array}{l}\text { Parambikulam Tiger } \\
\text { Reserve } \\
\text { Panthemthode, Silent } \\
\text { Valley NP }\end{array}$ & $\begin{array}{l}\text { Leelavathy \& } \\
\text { Ganesh } 2000\end{array}$ \\
\hline & & Thrissur & Vazhachal & $\begin{array}{l}\text { Leelavathy \& } \\
\text { Ganesh } 2000\end{array}$ \\
\hline & & Malappuram & $\begin{array}{l}\text { Nadukani, } \\
\text { Nilambur Calicut } \\
\text { University Campus }\end{array}$ & $\begin{array}{l}\text { Leelavathy \& } \\
\text { Ganesh } 2000\end{array}$ \\
\hline & & Malappuram & $\begin{array}{l}\text { Chandhakkunnu, } \\
\text { Nilambur }\end{array}$ & Mohanan 2011 \\
\hline & & Wayanad & Kuruva, Begur & Mohanan 2011 \\
\hline & & Ernakulam & $\begin{array}{l}\text { Iringole kavu, } \\
\text { Perumbavoor }\end{array}$ & Mohanan 2011 \\
\hline & & Thrissur & Kuthiran & Mohanan 2011 \\
\hline 46. & $\begin{array}{l}\text { Phellinus grenadensis } \\
\text { (Murrill) Ryvarden } 1972\end{array}$ & Ernakulam & $\begin{array}{l}\text { Iringole kavu, } \\
\text { Perumbavoor }\end{array}$ & Mohanan 2011 \\
\hline \multirow[t]{3}{*}{47.} & \multirow[t]{3}{*}{$\begin{array}{l}\text { Phellinus hoehnelii } \\
\text { (Bres.) Ryvarden } 1980\end{array}$} & Palakkad & $\begin{array}{l}\text { Kamathalamudi, } \\
\text { Parambikulam Tiger } \\
\text { Reserve }\end{array}$ & $\begin{array}{l}\text { Ganesh \& } \\
\text { Leelavathy } 1986\end{array}$ \\
\hline & & Palakkad & $\begin{array}{l}\text { Thunakadavu, } \\
\text { Parambikulam Tiger } \\
\text { Reserve }\end{array}$ & $\begin{array}{l}\text { Leelavathy \& } \\
\text { Ganesh } 2000\end{array}$ \\
\hline & & Wayanad & Kuruva, Begur & Mohanan 2011 \\
\hline \multirow[t]{2}{*}{48.} & \multirow[t]{2}{*}{$\begin{array}{l}\text { Phellinus nilgheriensis } \\
\text { (Mont.) G. Cunn. } 1965\end{array}$} & Wayanad & $\begin{array}{l}\text { Kuppadi, Sulthan } \\
\text { Bettery }\end{array}$ & $\begin{array}{l}\text { Leelavathy \& } \\
\text { Ganesh } 2000\end{array}$ \\
\hline & & Thrissur & Peechi & $\begin{array}{l}\text { Florence \& } \\
\text { Yesodharan } 2000\end{array}$ \\
\hline 49. & $\begin{array}{l}\text { Phellinus punctatiformis } \\
\text { (Murrill) Ryvarden } 1972\end{array}$ & Idukki & Mankulam & Mohanan 2011 \\
\hline \multirow[t]{5}{*}{50.} & \multirow[t]{5}{*}{$\begin{array}{l}\text { Phellinus rimosus (Berk.) } \\
\text { Pilát } 1940\end{array}$} & Malappuram & $\begin{array}{l}\text { Nilambur } \\
\text { Calicut University } \\
\text { Campus }\end{array}$ & $\begin{array}{l}\text { Leelavathy \& } \\
\text { Ganesh } 2000\end{array}$ \\
\hline & & Thrissur & Ayyanthole & $\begin{array}{l}\text { Leelavathy \& } \\
\text { Ganesh } 2000\end{array}$ \\
\hline & & Kottayam & Chakkumpuzha, Palai & $\begin{array}{l}\text { Leelavathy \& } \\
\text { Ganesh } 2000\end{array}$ \\
\hline & & Wayanad & Kuruva, Begur & Mohanan 2011 \\
\hline & & Idukki & Chinnar, Idukki & Mohanan 2011 \\
\hline 51. & $\begin{array}{l}\text { Phellinus robiniae } \\
\text { (Murrill) A. Ames } 1913\end{array}$ & Palakkad & Nelliyampathy & Mohanan 2011 \\
\hline 52. & $\begin{array}{l}\text { Phylloporia chrysites } \\
\text { (Berk.) Ryvarden } 1972\end{array}$ & Malappuram & $\begin{array}{l}\text { Calicut University } \\
\text { Campus }\end{array}$ & $\begin{array}{l}\text { Leelavathy \& } \\
\text { Ganesh } 2000\end{array}$ \\
\hline
\end{tabular}


Table 1 Continued.

\begin{tabular}{|c|c|c|c|c|}
\hline & Scientific Name & \multicolumn{2}{|c|}{ DISTRIBUTION } & \multirow[t]{2}{*}{ References } \\
\hline & & District & Locations & \\
\hline (III) & \multicolumn{4}{|c|}{ FAMILY : HYMENOCHAETACEAE } \\
\hline \multirow[t]{4}{*}{53.} & \multirow[t]{2}{*}{$\begin{array}{l}\text { Phellinus dependens } \\
\text { (Murrill) Ryvarden } 1972\end{array}$} & Ernakulam & Malayattoor & $\begin{array}{l}\text { Sankaran \& } \\
\text { Florence } 1995\end{array}$ \\
\hline & & Thrissur & Peechi WLS & $\begin{array}{l}\text { Florence \& } \\
\text { Yesodharan 1997, } \\
2000\end{array}$ \\
\hline & \multirow{2}{*}{$\begin{array}{l}\text { Tropicoporus dependens } \\
\text { (Murrill) L.W. Zhou, } \\
\text { Y.C. Dai \& Vlasák } 2015\end{array}$} & Thrissur & $\begin{array}{l}\text { Perumpara(W), } \\
\text { Malakkappara }\end{array}$ & $\begin{array}{l}\text { Leelavathy \& } \\
\text { Ganesh } 2000\end{array}$ \\
\hline & & Wayanad & Kathiyur reserve forest & $\begin{array}{l}\text { Leelavathy \& } \\
\text { Ganesh } 2000\end{array}$ \\
\hline (IV) & \multicolumn{4}{|c|}{ FAMILY: MERIPILACEAE } \\
\hline 54. & $\begin{array}{l}\text { Flaviporus minutisporus } \\
\text { (D.A. Reid, K.S. Thind \& } \\
\text { Chatr.) Ginns } 1980\end{array}$ & Wayanad & Chembra Hills, Kalpettta & Mohanan 2011 \\
\hline \multirow[t]{2}{*}{55.} & \multirow{2}{*}{$\begin{array}{l}\text { Gloeoporus } \\
\text { thelephoroides (Hook.) G. } \\
\text { Cunn. } 1965\end{array}$} & Wayanad & Muthanga & Mohanan 2011 \\
\hline & & Thiruvananthapuram & Arippa & Mohanan 2011 \\
\hline \multirow[t]{4}{*}{56.} & \multirow[t]{4}{*}{$\begin{array}{l}\text { Podoscypha venustula } \\
\text { (Speg.) D.A. Reid } 1965\end{array}$} & Ernakulam & $\begin{array}{l}\text { Iringole Kavu, } \\
\text { Permbavoor }\end{array}$ & Mohanan 2011 \\
\hline & & Wayanad & Muthanga & Mohanan 2011 \\
\hline & & Wayanad & Kuruva, Begur & Mohanan 2011 \\
\hline & & Idukki & Anamudishola, Munnar & Mohanan 2011 \\
\hline \multirow[t]{9}{*}{57.} & \multirow[t]{9}{*}{$\begin{array}{l}\text { Rigidoporus lineatus } \\
\text { (Pers.) Ryvarden } 1972\end{array}$} & Thrissur & $\begin{array}{l}\text { Peechi Palappilly Estate } \\
\text { Perumpara (W), } \\
\text { Malakkapara, Sholayar }\end{array}$ & $\begin{array}{l}\text { Leelavathy \& } \\
\text { Ganesh } 2000\end{array}$ \\
\hline & & Ernakulam & Malayattoor & $\begin{array}{l}\text { Sankaran \& } \\
\text { Florence } 1995 \\
\end{array}$ \\
\hline & & Thrissur & Peechi WLS & $\begin{array}{l}\text { Florence \& } \\
\text { Yesodharan 1997, } \\
2000\end{array}$ \\
\hline & & Thrissur & $\begin{array}{l}\text { Peechi Palappilly Estate } \\
\text { Perumpara (W), } \\
\text { Malakkapara, Sholayar }\end{array}$ & $\begin{array}{l}\text { Leelavathy \& } \\
\text { Ganesh } 2000\end{array}$ \\
\hline & & Malappuram & $\begin{array}{l}\text { Nedumkayam, } \\
\text { Nilambur } \\
\text { Kanjiramkadavu, } \\
\text { Nilambur }\end{array}$ & $\begin{array}{l}\text { Leelavathy \& } \\
\text { Ganesh } 2000\end{array}$ \\
\hline & & Wayanad & $\begin{array}{l}\text { Chethalayam, } \\
\text { Noolpuzha, Muthanga }\end{array}$ & $\begin{array}{l}\text { Leelavathy \& } \\
\text { Ganesh } 2000\end{array}$ \\
\hline & & Calicut & Pokkunnu & $\begin{array}{l}\text { Leelavathy \& } \\
\text { Ganesh } 2000\end{array}$ \\
\hline & & Palakkad & $\begin{array}{l}\text { Pathamthde, Silent } \\
\text { Valley NP }\end{array}$ & $\begin{array}{l}\text { Leelavathy \& } \\
\text { Ganesh } 2000\end{array}$ \\
\hline & & Wayanad & Kuppadi, Sultan Battery & $\begin{array}{l}\text { Leelavathy \& } \\
\text { Ganesh } 2000\end{array}$ \\
\hline \multirow[t]{5}{*}{58.} & \multirow[t]{5}{*}{$\begin{array}{l}\text { Rigidoporus microporus } \\
\text { (Sw.) Overeem } 1924\end{array}$} & Thrissur & $\begin{array}{l}\text { Perumpara }(\mathrm{W}), \\
\text { Malakkapara, } \\
\text { Sholayar }\end{array}$ & $\begin{array}{l}\text { Leelavathy \& } \\
\text { Ganesh } 2000\end{array}$ \\
\hline & & Wayanad & Begur & $\begin{array}{l}\text { Leelavathy \& } \\
\text { Ganesh } 2000\end{array}$ \\
\hline & & Thiruvananthapuram & Kallar, Ponmudi & Mohanan 2011 \\
\hline & & Thiruvananthapuram & Arippa & Mohanan 2011 \\
\hline & & Malappuram & Nadukani, Nilambur & Mohanan 2011 \\
\hline
\end{tabular}


Table 1 Continued.

\begin{tabular}{|c|c|c|c|c|}
\hline & Scientific Name & \multicolumn{2}{|c|}{ DISTRIBUTION } & \multirow[t]{2}{*}{ References } \\
\hline & & District & Locations & \\
\hline (IV) & \multicolumn{4}{|c|}{ FAMILY: MERIPILACEAE } \\
\hline & & Ernakulam & $\begin{array}{l}\text { Iringole kavu, } \\
\text { Perumbavoor }\end{array}$ & Mohanan 2011 \\
\hline \multirow[t]{5}{*}{59.} & \multirow[t]{5}{*}{$\begin{array}{l}\text { Rigidoporus ulmarius } \\
\text { (Sowerby) Imazeki } 1952\end{array}$} & Wayanad & $\begin{array}{l}\text { North Pathiri Reserve } \\
\text { Forest, Perikaloor }\end{array}$ & $\begin{array}{l}\text { Leelavathy \& } \\
\text { Ganesh } 2000\end{array}$ \\
\hline & & Thrissur & Malakkapara, Sholayar & $\begin{array}{l}\text { Leelavathy \& } \\
\text { Ganesh } 2000\end{array}$ \\
\hline & & Idukki & $\begin{array}{l}\text { Mannavan shola, } \\
\text { Munnar }\end{array}$ & Mohanan 2011 \\
\hline & & Wayanad & Chethalayam & Mohanan 2011 \\
\hline & & Idukki & Vattavada, Munnar & Mohanan 2011 \\
\hline 60. & $\begin{array}{l}\text { Rigidoporus vinctus } \\
\text { (Berk.) Ryvarden } 1972\end{array}$ & Malappuram & $\begin{array}{l}\text { Kanjirakadavu, } \\
\text { Nilambur }\end{array}$ & $\begin{array}{l}\text { Leelavathy \& } \\
\text { Ganesh } 2000\end{array}$ \\
\hline (V) & \multicolumn{4}{|l|}{ FAMILY: MERULIACEAE } \\
\hline 61. & $\begin{array}{l}\text { Abortiporus biennis } \\
\text { (Bull.) Singer } 1944\end{array}$ & Wayanad & Sultan Battery & $\begin{array}{l}\text { Leelavathy \& } \\
\text { Ganesh } 2000\end{array}$ \\
\hline 62. & $\begin{array}{l}\text { Bjerkandera adusta } \\
\text { (Willd.) P. Karst. } 1879\end{array}$ & Idukki & Anamudishola, Munnar & Mohanan 2011 \\
\hline \multirow[t]{7}{*}{63.} & \multirow{7}{*}{$\begin{array}{l}\text { Flavodon flavus } \\
\text { (Klotzsch) Ryvarden } \\
1973\end{array}$} & Ernakulam & Malayattoor & $\begin{array}{l}\text { Sankaran \& } \\
\text { Florence } 1995\end{array}$ \\
\hline & & Thrissur & Peechi WLS & $\begin{array}{l}\text { Florence \& } \\
\text { Yesodharan 1997, } \\
2000\end{array}$ \\
\hline & & Malappuram & $\begin{array}{l}\text { Calicut University } \\
\text { Campus }\end{array}$ & $\begin{array}{l}\text { Leelavathy \& } \\
\text { Ganesh } 2000\end{array}$ \\
\hline & & Wayanad & Wayanad Reserve forest & $\begin{array}{l}\text { Leelavathy \& } \\
\text { Ganesh } 2000\end{array}$ \\
\hline & & Thrissur & Peechi & Mohanan 2011 \\
\hline & & Wayanad & Manikunnumala & Mohanan 2011 \\
\hline & & Wayanad & Kalpetta & Mohanan 2011 \\
\hline \multirow[t]{2}{*}{64.} & \multirow[t]{2}{*}{$\begin{array}{l}\text { Irpex lacteus (Fr.) Fr. } \\
1828\end{array}$} & Wayanad & $\begin{array}{l}\text { Karadimala, Brahmagiri, } \\
\text { Thirunelli }\end{array}$ & $\begin{array}{l}\text { Leelavathy \& } \\
\text { Ganesh } 2000\end{array}$ \\
\hline & & Kollam & Kulathupuzha & Mohanan 2011 \\
\hline 65. & $\begin{array}{l}\text { Junghuhnia crustacea } \\
\text { (Jungh.) Ryvarden } 1972\end{array}$ & Wayanad & Chembra peak, Meppadi & $\begin{array}{l}\text { Leelavathy \& } \\
\text { Ganesh } 2000\end{array}$ \\
\hline (VI) & \multicolumn{4}{|c|}{ FAMILY: PHANEROCHAETACEAE } \\
\hline \multirow[t]{6}{*}{66.} & \multirow{6}{*}{$\begin{array}{l}\text { Oxychaete cervinogilva } \\
\text { (Jungh.) Miettinen } 2016\end{array}$} & Idukki & Mankulam & Mohanan 2011 \\
\hline & & Ernakulam & $\begin{array}{l}\text { Iringole Kavu, } \\
\text { Permbavoor }\end{array}$ & Mohanan 2011 \\
\hline & & Pathanamthitta & Konni & Mohanan 2011 \\
\hline & & Malappuram & Nilambur & $\begin{array}{l}\text { Leelavathy \& } \\
\text { Ganesh } 2000\end{array}$ \\
\hline & & Kannur & Pazhayangadi & $\begin{array}{l}\text { Leelavathy and } \\
\text { Ganesh } 2000\end{array}$ \\
\hline & & Wayanad & $\begin{array}{l}\text { Vellarimala, Chembra } \\
\text { peak, Meppadi }\end{array}$ & $\begin{array}{l}\text { Leelavathy \& } \\
\text { Ganesh } 2000\end{array}$ \\
\hline (VII) & \multicolumn{4}{|c|}{ FAMILY: POLYPORACEAE } \\
\hline \multirow[t]{3}{*}{67.} & \multirow{3}{*}{$\begin{array}{l}\text { Loweporus fuscopurpureus } \\
\text { (Pers.) Ryvarden } 1980 \\
\text { Abundisporus } \\
\text { fuscopurpureus (Pers.) } \\
\text { Ryvarden } 1999\end{array}$} & Ernakulam & Malayattoor & $\begin{array}{l}\text { Sankaran \& } \\
\text { Florence } 1995\end{array}$ \\
\hline & & Thrissur & Peechi & $\begin{array}{l}\text { Florence \& } \\
\text { Yesodaran 1997, } \\
2000\end{array}$ \\
\hline & & Thrissur & Sholayar & $\begin{array}{l}\text { Leelavathy \& } \\
\text { Ganesh } 2000\end{array}$ \\
\hline
\end{tabular}


Table 1 Continued.

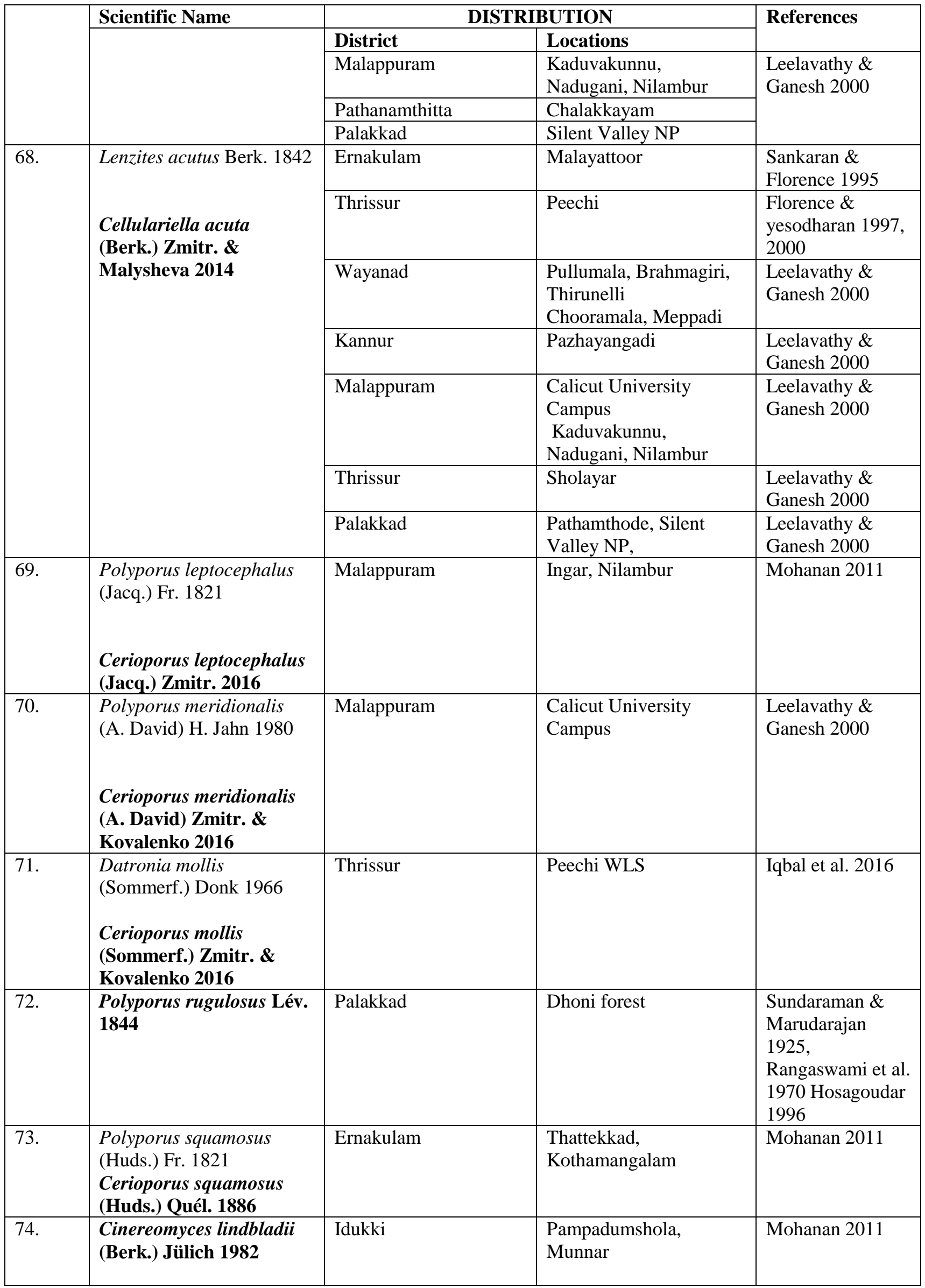


Table 1 Continued.

\begin{tabular}{|c|c|c|c|c|}
\hline & Scientific Name & \multicolumn{2}{|c|}{ DISTRIBUTION } & \multirow[t]{2}{*}{ References } \\
\hline & & District & Locations & \\
\hline (VII) & \multicolumn{4}{|c|}{ FAMILY: POLYPORACEAE } \\
\hline 75. & $\begin{array}{l}\text { Coriolopsis occidentalis } \\
\text { (Klotzsch) Murrill } 1905\end{array}$ & Palakkad & Nelliyampathy & Mohanan 2011 \\
\hline \multirow[t]{9}{*}{76.} & \multirow[t]{9}{*}{$\begin{array}{l}\text { Earliella scabrosa (Pers.) } \\
\text { Gilb. \& Ryvarden } 1985 \\
\\
\text { Trametes scabrosa (Pers.) } \\
\text { G. Cunn. } 1965\end{array}$} & Malappuram & $\begin{array}{l}\text { Calicut University } \\
\text { Campus Cannoly's teak } \\
\text { plantation, Nilambur } \\
\text { Kanjiramkadavu, } \\
\text { Nilambur } \\
\end{array}$ & $\begin{array}{l}\text { Leelavathy \& } \\
\text { Ganesh } 2000\end{array}$ \\
\hline & & Kottayam & $\begin{array}{l}\text { Kumarakam Bird } \\
\text { Sanctuary }\end{array}$ & $\begin{array}{l}\text { Leelavathy \& } \\
\text { Ganesh } 2000\end{array}$ \\
\hline & & Calicut & Thamarassery & $\begin{array}{l}\text { Leelavathy \& } \\
\text { Ganesh } 2000\end{array}$ \\
\hline & & Wayanad & Kuppadi, Sultan Battery & $\begin{array}{l}\text { Leelavathy \& } \\
\text { Ganesh } 2000\end{array}$ \\
\hline & & Ernakulam & $\begin{array}{l}\text { Iringole Kavu, } \\
\text { Perumbavoor }\end{array}$ & Mohanan 2011 \\
\hline & & Idukki & Kulamavu & Mohanan, 2011 \\
\hline & & Malappuram & $\begin{array}{l}\text { Chandhakunnu, } \\
\text { Nilambur }\end{array}$ & Mohanan 2011 \\
\hline & & Thiruvananthapuram & Ammayambalam & Mohanan 2011 \\
\hline & & Thiruvananthapuram & Arippa & Mohanan 2011 \\
\hline 77. & $\begin{array}{l}\text { Echinochaete ruficeps } \\
\text { (Berk. \& Broome) } \\
\text { Ryvarden } 1972\end{array}$ & Polyporaceae & Nadukani, Nilambur & Mohanan 2011 \\
\hline \multirow[t]{6}{*}{78.} & \multirow{6}{*}{$\begin{array}{l}\text { Favolus brasiliensis (Fr.) } \\
\text { Fr. } 1830 \\
\text { Favolus tenuiculus } \mathbf{P} \text {. } \\
\text { Beauv. } 1806\end{array}$} & Ernakulam & Malayattoor & $\begin{array}{l}\text { Sankaran \& } \\
\text { Florence } 1995\end{array}$ \\
\hline & & Malappuram & $\begin{array}{l}\text { Calicut University } \\
\text { Campus Nilambur }\end{array}$ & $\begin{array}{l}\text { Leelavathy \& } \\
\text { Ganesh } 2000\end{array}$ \\
\hline & & Palakkad & $\begin{array}{l}\text { Panthemthode, Silent } \\
\text { Valley NP }\end{array}$ & $\begin{array}{l}\text { Leelavathy \& } \\
\text { Ganesh } 2000\end{array}$ \\
\hline & & Wayanad & $\begin{array}{l}\text { Mundakal Forest, } \\
\text { Meppadi }\end{array}$ & $\begin{array}{l}\text { Leelavathy \& } \\
\text { Ganesh } 2000\end{array}$ \\
\hline & & Thrissur & $\begin{array}{l}\text { Perumpara }(\mathrm{W}), \\
\text { Sholayar }\end{array}$ & $\begin{array}{l}\text { Leelavathy \& } \\
\text { Ganesh } 2000\end{array}$ \\
\hline & & Thrissur & Peechi & $\begin{array}{l}\text { Florence \& } \\
\text { Yesodharan 2000, } \\
\text { Mohanan 2011 }\end{array}$ \\
\hline \multirow[t]{2}{*}{79.} & \multirow{2}{*}{$\begin{array}{l}\text { Phellinus troyanus } \\
\text { (Murrill) Bondartseva \& S. } \\
\text { Herrera } 1980 \\
\\
\text { Fomes extensus (Lév.) } \\
\text { Cooke 1885 }\end{array}$} & Palakkad & $\begin{array}{l}\text { Vengoli peak, } \\
\text { Parambikulam Tiger } \\
\text { Rereve }\end{array}$ & $\begin{array}{l}\text { Leelavathy \& } \\
\text { Ganesh } 2000\end{array}$ \\
\hline & & Thrissur & Malakkappara & $\begin{array}{l}\text { Leelavathy \& } \\
\text { Ganesh } 2000\end{array}$ \\
\hline \multirow[t]{3}{*}{80.} & \multirow{2}{*}{$\begin{array}{l}\text { Phellinus pseudosenex } \\
\text { (Murrill) Bondartseva \& S. } \\
\text { Herrera } 1980\end{array}$} & Thrissur & $\begin{array}{l}\text { Perumpara(W), } \\
\text { Malakkappara }\end{array}$ & $\begin{array}{l}\text { Leelavathy \& } \\
\text { Ganesh } 2000\end{array}$ \\
\hline & & Palakkad & $\begin{array}{l}\text { Thunakadavu, } \\
\text { Parambikulam Tiger } \\
\text { Reserve }\end{array}$ & $\begin{array}{l}\text { Leelavathy \& } \\
\text { Ganesh } 2000\end{array}$ \\
\hline & $\begin{array}{l}\text { Fomes pseudosenex } \\
\text { (Murrill) Sacc. \& Trotter } \\
1912\end{array}$ & Thrissur & Kuthiran & Mohanan 2011 \\
\hline
\end{tabular}


Table 1 Continued.

\begin{tabular}{|c|c|c|c|c|}
\hline & Scientific Name & \multicolumn{2}{|l|}{ DISTRIBUTION } & \multirow[t]{2}{*}{ References } \\
\hline & & District & Locations & \\
\hline (VII) & \multicolumn{4}{|c|}{ FAMILY: POLYPORACEAE } \\
\hline \multirow[t]{4}{*}{81.} & \multirow[t]{2}{*}{$\begin{array}{l}\text { Coriolopsis caperata } \\
\text { (Berk.) Murrill } 1908\end{array}$} & Malappuram & $\begin{array}{l}\text { Kaduvakunnu, Nadigani, } \\
\text { Nilambur }\end{array}$ & $\begin{array}{l}\text { Leelavathy \& } \\
\text { Ganesh } 2000\end{array}$ \\
\hline & & Palakkad & Silent Valley NP & $\begin{array}{l}\text { Leelavathy \& } \\
\text { Ganesh } 2000\end{array}$ \\
\hline & \multirow{2}{*}{$\begin{array}{l}\text { Funalia caperata (Berk.) } \\
\text { Zmitr. \& Malysheva } \\
2013\end{array}$} & Wayanad & $\begin{array}{l}\text { Kanoth Reserve forest, } \\
\text { Boonthakkal, Begur }\end{array}$ & $\begin{array}{l}\text { Leelavathy \& } \\
\text { Ganesh } 2000\end{array}$ \\
\hline & & Wayanad & Periya & Mohanan 2011 \\
\hline \multirow[t]{2}{*}{82.} & \multirow{2}{*}{$\begin{array}{l}\text { Coriolopsis sanguinaria } \\
\text { (Klotzsch) Teng } 1963 \\
\text { Funalia sanguinaria } \\
\text { (Klotzsch) Zmitr. \& } \\
\text { Malysheva 2013 }\end{array}$} & Malappuram & Nadigani, Nilambur & $\begin{array}{l}\text { Leelavathy \& } \\
\text { Ganesh } 2000\end{array}$ \\
\hline & & Palakkad & Silent Valley NP & $\begin{array}{l}\text { Leelavathy \& } \\
\text { Ganesh } 2000\end{array}$ \\
\hline \multirow[t]{9}{*}{83.} & \multirow[t]{9}{*}{$\begin{array}{l}\text { Hexagonia tenuis (Fr.) } \\
\text { Fr. } 1838\end{array}$} & Ernakulam & Malayattoor & $\begin{array}{l}\text { Sankaran \& } \\
\text { Florence } 1995 \\
\end{array}$ \\
\hline & & Thrissur & Peechi & $\begin{array}{l}\text { Florence \& } \\
\text { Yesodharan 1997, } \\
2000\end{array}$ \\
\hline & & Malappuram & $\begin{array}{l}\text { Calicut University } \\
\text { Campus }\end{array}$ & $\begin{array}{l}\text { Leelavathy \& } \\
\text { Ganesh } 2000\end{array}$ \\
\hline & & Kannur & Pazhayangadi & $\begin{array}{l}\text { Leelavathy \& } \\
\text { Ganesh } 2000\end{array}$ \\
\hline & & Wayanad & $\begin{array}{l}\text { Karadimala, Brahmagiri, } \\
\text { Thirunelli }\end{array}$ & $\begin{array}{l}\text { Leelavathy \& } \\
\text { Ganesh } 2000\end{array}$ \\
\hline & & Malappuram & Nilambur & Mohanan 2011 \\
\hline & & Idukki & Anamudishola, Munnar & Mohanan 2011 \\
\hline & & Ernakulam & $\begin{array}{l}\text { Iringole Kavu, } \\
\text { Perumbavoor }\end{array}$ & Mohanan 2011 \\
\hline & & Wayanad & Kuruva, Begur & Mohanan 2011 \\
\hline 84. & $\begin{array}{l}\text { Trametes lactinea (Berk.) } \\
\text { Sacc. } 1888 \\
\text { Leiotrametes lactinea } \\
\text { (Berk.) Welti \& Courtec. } \\
\mathbf{2 0 1 2}\end{array}$ & Wayanad & $\begin{array}{l}\text { Karadimala, Brahmagiri, } \\
\text { Thirunelli }\end{array}$ & $\begin{array}{l}\text { Leelavathy \& } \\
\text { Ganesh } 2000\end{array}$ \\
\hline \multirow[t]{8}{*}{85.} & \multirow[t]{8}{*}{$\begin{array}{l}\text { Polyporus arcularius } \\
\text { (Batsch) Fr. } 1821\end{array}$} & Ernakulam & Malayattoor & $\begin{array}{l}\text { Sankaran \& } \\
\text { Florence } 1995\end{array}$ \\
\hline & & Thrissur & Peechi & $\begin{array}{l}\text { Florence \& } \\
\text { Yesodharan 1997, } \\
2000\end{array}$ \\
\hline & & Malappuram & $\begin{array}{l}\text { Calicut University } \\
\text { Campus }\end{array}$ & $\begin{array}{l}\text { Leelavathy \& } \\
\text { Ganesh } 2000\end{array}$ \\
\hline & & Wayanad & $\begin{array}{l}\text { Mundakai, Meppadi } \\
\text { Karadimala, Brahmagiri, } \\
\text { Thirunelli } \\
\text { Chooramala, Meppadi }\end{array}$ & $\begin{array}{l}\text { Leelavathy \& } \\
\text { Ganesh } 2000\end{array}$ \\
\hline & & Palakkad & $\begin{array}{l}\text { Tunakadavu, } \\
\text { Parmbikulam Tiger } \\
\text { Reserve }\end{array}$ & $\begin{array}{l}\text { Leelavathy \& } \\
\text { Ganesh } 2000\end{array}$ \\
\hline & & Malappuram & $\begin{array}{l}\text { Chandhakkunnu, } \\
\text { Nilambur }\end{array}$ & Mohanan 2011 \\
\hline & & Idukki & Munnar, Devikulam & Mohanan 2011 \\
\hline & & Thiruvananthapuram & Arippa & Mohanan 2011 \\
\hline
\end{tabular}


Table 1 Continued.

\begin{tabular}{|c|c|c|c|c|}
\hline & Scientific Name & \multicolumn{2}{|l|}{ DISTRIBUTION } & \multirow[t]{2}{*}{ References } \\
\hline & & District & Locations & \\
\hline (VII) & \multicolumn{4}{|c|}{ FAMILY: POLYPORACEAE } \\
\hline 86. & $\begin{array}{l}\text { Lentinus bambusinus } \\
\text { T.K.A. Kumar \& } \\
\text { Manim., Mycotaxon 92: } \\
119 \text { (2005) }\end{array}$ & Malappuram & - & $\begin{array}{l}\text { Kumar \& } \\
\text { Manimohan } 2005\end{array}$ \\
\hline 87. & $\begin{array}{l}\text { Lentinus caespiticola Pat. } \\
\text { \& Har. } 1900\end{array}$ & Malappuram & - & $\begin{array}{l}\text { Manimohan \& } \\
\text { Leelavathy } 1995 \\
\{\text { as } L . \\
\text { caespiticola var. } \\
\text { asiaticus Manim. } \\
\text { Leelav. ^\}, } \\
\text { Manimohan et al. } \\
2004\end{array}$ \\
\hline \multirow[t]{2}{*}{88.} & \multirow{2}{*}{$\begin{array}{l}\text { Lentinus dicholamellatus } \\
\text { Manim. } 2004\end{array}$} & Malappuram & Poonthanam & Mohanan 2011 \\
\hline & & $\begin{array}{l}\text { Thiruvananthapuram, } \\
\text { Wayanad }\end{array}$ & - & $\begin{array}{l}\text { Manimohan et al. } \\
\text { 2004, Pradeep \& } \\
\text { Vrinda 2007, } \\
\text { Varghese et al. } \\
2010\end{array}$ \\
\hline 89. & $\begin{array}{l}\text { Lentinus giganteus Berk. } \\
1847\end{array}$ & $\begin{array}{l}\text { Thiruvananthapuram, } \\
\text { Malappuram, Kollam }\end{array}$ & Palode & $\begin{array}{l}\text { Joseph et al. } \\
\text { 1995, Manimohan } \\
\text { et al. 2004, } \\
\text { Pradeep \& Vrinda } \\
\text { 2007, Mohanan } \\
\text { 2011. Hosagouder } \\
\text { et al } 1996\end{array}$ \\
\hline 90. & $\begin{array}{l}\text { Lentinus patulus Lév. } \\
1846\end{array}$ & Malappuram & Nadukani, Nilambur & Mohanan 2011 \\
\hline \multirow[t]{2}{*}{91.} & \multirow[t]{2}{*}{$\begin{array}{l}\text { Lentinus polychrous Lév. } \\
1844\end{array}$} & Thiruvananthapuram & - & $\begin{array}{l}\text { Manimohan et al. } \\
2004\end{array}$ \\
\hline & & Ernakulam & $\begin{array}{l}\text { Iringole Kavu, } \\
\text { Perumbavoor }\end{array}$ & Mohanan 2011 \\
\hline \multirow[t]{2}{*}{92.} & \multirow[t]{2}{*}{$\begin{array}{l}\text { Lentinus sajor-caju (Fr.) } \\
\text { Fr. } 1838\end{array}$} & Thiruvananthappuram & $\begin{array}{l}\text { Ammayambalam, } \\
\text { Arippa Kochupilakkode, } \\
\text { Arippa }\end{array}$ & Mohanan 2011 \\
\hline & & $\begin{array}{l}\text { Kottayam, } \\
\text { Thiruvananthapuram } \\
\text { Wayanad, Kollam, } \\
\text { Thrissur }\end{array}$ & - & $\begin{array}{l}\text { Sathe \& Daniel } \\
\text { 1980, Florence } \\
\text { 2004, Manimohan } \\
\text { et al. 2004, } \\
\text { Pradeep \& Vrinda } \\
\text { 2007, Varghese et } \\
\text { al. 2010, } \\
\text { Mohanan 2011. } \\
\text { As Pleurotus } \\
\text { sajor-caju (Fr.) } \\
\text { Singer in Geetha } \\
\text { et al. 1989, } \\
\text { Florence \& } \\
\text { Balasundaran } \\
\text { 2000, Ouseph et } \\
\text { al. 2001, Florence } \\
\text { 2004 }\end{array}$ \\
\hline
\end{tabular}


Table 1 Continued.

\begin{tabular}{|c|c|c|c|c|}
\hline & Scientific Name & \multicolumn{2}{|c|}{ DISTRIBUTION } & \multirow[t]{2}{*}{ References } \\
\hline & & District & Locations & \\
\hline (VII) & \multicolumn{4}{|c|}{ FAMILY: POLYPORACEAE } \\
\hline \multirow[t]{6}{*}{93.} & \multirow{6}{*}{$\begin{array}{l}\text { Lentinus squarrosulus } \\
\text { Mont. } 1842\end{array}$} & Thrissur & Peechi & $\begin{array}{l}\text { Florence \& } \\
\text { Yesodharan } 2000\end{array}$ \\
\hline & & Wayanad & Thamarassery churam, & Mohanan 2011 \\
\hline & & Thiruvananthapuram & $\begin{array}{l}\text { Ammayambalam, } \\
\text { Arippa }\end{array}$ & Mohanan 2011 \\
\hline & & Malappuram & $\begin{array}{l}\text { Chandhakkunnu, } \\
\text { Nilambur }\end{array}$ & Mohanan 2011 \\
\hline & & Ernakulam & $\begin{array}{l}\text { Iringole Kavu, } \\
\text { Perumbavoor }\end{array}$ & Mohanan 2011 \\
\hline & & $\begin{array}{l}\text { Ernakulam, Thrissur, } \\
\text { Thiruvananthapuram, } \\
\text { Palakkad, Malappuram, } \\
\text { Wayanad, Alappuzha, } \\
\text { Kollam }\end{array}$ & - & $\begin{array}{l}\text { Sharma et al. } \\
\text { 1985, Sankran et } \\
\text { al. 1995, Florence } \\
\text { \& Yesodharan } \\
\text { 2000, Florence } \\
\text { 2004, Manimohan } \\
\text { et al. 2004, } \\
\text { Pradeep \& Vrinda } \\
\text { 2007, Varghese et } \\
\text { al. 2010, } \\
\text { Mohanan } 2011\end{array}$ \\
\hline 94. & $\begin{array}{l}\text { Lentinus strigosus Fr. } \\
1825\end{array}$ & $\begin{array}{l}\text { Wayanad, Kannur, } \\
\text { Malappuram }\end{array}$ & - & $\begin{array}{l}\text { Manimohan et al. } \\
\text { 2004, Mohanan } \\
\text { 2011 }\end{array}$ \\
\hline \multirow[t]{2}{*}{95.} & \multirow[t]{2}{*}{$\begin{array}{l}\text { Lentinus tigrinus (Bull.) } \\
\text { Fr. } 1825\end{array}$} & Ernakulam & $\begin{array}{l}\text { Iringole Kavu, } \\
\text { Perumbavoor }\end{array}$ & Mohanan 2011 \\
\hline & & Wayanad & Muthanga & Mohanan 2011 \\
\hline \multirow[t]{2}{*}{96.} & \multirow[t]{2}{*}{$\begin{array}{l}\text { Lentinus tricholoma } \\
\text { (Mont.) Zmitr. } 2010\end{array}$} & Malappuram & $\begin{array}{l}\text { Calicut University } \\
\text { Campus }\end{array}$ & $\begin{array}{l}\text { Leelavathy \& } \\
\text { Ganesh } 2000\end{array}$ \\
\hline & & Malappuram & Karulai, Nilambur & Mohanan 2011 \\
\hline 97. & $\begin{array}{l}\text { Lentinus tuber-regium } \\
\text { (Fr.) Fr. } 1836\end{array}$ & Thiruvananthapuram & - & $\begin{array}{l}\text { Geetha et al. } \\
\text { 2002, Florence } \\
2004 \text { (as } \\
\text { Pleurotus tuber- } \\
\text { regium (Rumph. } \\
\text { ex Fr.) Singer }\end{array}$ \\
\hline \multirow[t]{2}{*}{98.} & \multirow[t]{2}{*}{$\begin{array}{l}\text { Lenzites betulinus (L.) Fr. } \\
1838\end{array}$} & Wayanad & $\begin{array}{l}\text { Vellarimala, Chembra } \\
\text { peak, Meppadi }\end{array}$ & $\begin{array}{l}\text { Leelavathy \& } \\
\text { Ganesh 2000, } \\
\text { Florence 2004 }\end{array}$ \\
\hline & & Idukki & $\begin{array}{l}\text { Pampadumshola, } \\
\text { Munnar }\end{array}$ & Mohanan 2011 \\
\hline \multirow[t]{2}{*}{99.} & \multirow[t]{2}{*}{$\begin{array}{l}\text { Loweporus tephroporus } \\
\text { (Mont.) Ryvarden } 1980\end{array}$} & Wayanad & $\begin{array}{l}\text { Kuppadi, Sultan Battery } \\
\text { Noolpuzha, Muthanga }\end{array}$ & $\begin{array}{l}\text { Leelavathy \& } \\
\text { Ganesh } 2000\end{array}$ \\
\hline & & Thrissur & Poothole & $\begin{array}{l}\text { Leelavathy \& } \\
\text { Ganesh } 2000\end{array}$ \\
\hline 100. & $\begin{array}{l}\text { Nigroporus niger (Berk.) } \\
\text { Ryvarden } 1972 \\
\text { Melanoporia nigra } \\
\text { (Berk.) Murrill (1907) }\end{array}$ & Malappuram & $\begin{array}{l}\text { Kanjirakadavu, } \\
\text { Amarambala, Nilambur }\end{array}$ & $\begin{array}{l}\text { Leelavathy \& } \\
\text { Ganesh } 2000\end{array}$ \\
\hline
\end{tabular}


Table 1 Continued.

\begin{tabular}{|c|c|c|c|c|}
\hline & Scientific Name & \multicolumn{2}{|c|}{ DISTRIBUTION } & \multirow[t]{2}{*}{ References } \\
\hline & & District & Locations & \\
\hline (VII) & \multicolumn{4}{|c|}{ FAMILY: POLYPORACEAE } \\
\hline \multirow[t]{8}{*}{101.} & \multirow[t]{8}{*}{$\begin{array}{l}\text { Microporellus obovatus } \\
\text { (Jungh.) Ryvarden } 1972\end{array}$} & Ernakulam & Malayattoor & $\begin{array}{l}\text { Sankaran \& } \\
\text { Florence } 1995\end{array}$ \\
\hline & & Thrissur & Peechi & $\begin{array}{l}\text { Florence \& } \\
\text { Yesodharan, } 1997\end{array}$ \\
\hline & & Malappuram & $\begin{array}{l}\text { Kanjirakadavu, } \\
\text { Nilambur }\end{array}$ & $\begin{array}{l}\text { Leelavathy \& } \\
\text { Ganesh } 2000\end{array}$ \\
\hline & & Thrissur & Malakkappara, Sholayar & $\begin{array}{l}\text { Leelavathy \& } \\
\text { Ganesh } 2000\end{array}$ \\
\hline & & Wayanad & $\begin{array}{l}\text { Vellarimala, Chembra } \\
\text { peak, Meppadi }\end{array}$ & $\begin{array}{l}\text { Leelavathy \& } \\
\text { Ganesh } 2000\end{array}$ \\
\hline & & Malappuram & Nadukani, Nilambur, & Mohanan 2011 \\
\hline & & Malappuram & $\begin{array}{l}\text { Chandhakkunnu, } \\
\text { Nilambur }\end{array}$ & Mohanan 2011 \\
\hline & & Ernakulam & $\begin{array}{l}\text { Iringole Kavu, } \\
\text { Perumbavoor }\end{array}$ & Mohanan 2011 \\
\hline \multirow[t]{3}{*}{102.} & \multirow{3}{*}{$\begin{array}{l}\text { Microporellus } \\
\text { violaceocinerascens } \\
\text { (Petch) A. David \& } \\
\text { Rajchenb. } 1985 \\
\end{array}$} & Thrissur & Peechi & Mohanan 2011 \\
\hline & & Wayanad & Kuppadi & Mohanan 2011 \\
\hline & & Wayanad & Kuruva, Begur & Mohanan 2011 \\
\hline \multirow[t]{11}{*}{103.} & \multirow{11}{*}{$\begin{array}{l}\text { Microporus affinis } \\
\text { (Blume \& T. Nees) } \\
\text { Kuntze } 1898\end{array}$} & Ernakulam & Malayattoor & $\begin{array}{l}\text { Sankaran \& } \\
\text { Florence } 1995\end{array}$ \\
\hline & & Thrissur & Peechi & $\begin{array}{l}\text { Florence \& } \\
\text { Yesodharan 1997, } \\
2000\end{array}$ \\
\hline & & Wayanad & $\begin{array}{l}\text { Choopramala, Meppadi } \\
\text { Kanoth Thirunelli } \\
\text { Noolpuzha, Muthanga } \\
\text { Periya Reserve Forest }\end{array}$ & $\begin{array}{l}\text { Leelavathy \& } \\
\text { Ganesh } 2000\end{array}$ \\
\hline & & Palakkad & $\begin{array}{l}\text { Pantamthode, Silent } \\
\text { Valley NP } \\
\text { Vengoli Peak, } \\
\text { parambikulam, }\end{array}$ & $\begin{array}{l}\text { Leelavathy \& } \\
\text { Ganesh } 2000\end{array}$ \\
\hline & & Malappuram & $\begin{array}{l}\text { Kaduvakunnu, } \\
\text { Nadugani, Nilambur }\end{array}$ & $\begin{array}{l}\text { Leelavathy \& } \\
\text { Ganesh } 2000 \\
\end{array}$ \\
\hline & & Thrissur & Vettilappara, Sholayar & $\begin{array}{l}\text { Leelavathy \& } \\
\text { Ganesh } 2000\end{array}$ \\
\hline & & Kasaragod & $\begin{array}{l}\text { Kerala- Mysore state } \\
\text { boundary }\end{array}$ & $\begin{array}{l}\text { Leelavathy \& } \\
\text { Ganesh } 2000\end{array}$ \\
\hline & & Alappuzha & - & $\begin{array}{l}\text { Leelavathy \& } \\
\text { Ganesh } 2000\end{array}$ \\
\hline & & Kollam & Arippa, Kulathupuzha & Mohanan 2011 \\
\hline & & Kollam & Nadukani, Kulathupuzha & Mohanan 2011 \\
\hline & & Idukki & Mannavanshola, Munnar & Mohanan 2011 \\
\hline \multirow[t]{4}{*}{104.} & \multirow[t]{4}{*}{$\begin{array}{l}\text { Microporus xanthopus } \\
\text { (Fr.) Kuntze } 1898\end{array}$} & Ernakulam & Malayattoor & $\begin{array}{l}\text { Sankaran \& } \\
\text { Florence } 1995\end{array}$ \\
\hline & & Thrissur & Peechi & $\begin{array}{l}\text { Florence \& } \\
\text { Yesodharan 1997, } \\
2000\end{array}$ \\
\hline & & Ernakulam & Malayattoor & $\begin{array}{l}\text { Sankaran \& } \\
\text { Florence } 1995 \\
\end{array}$ \\
\hline & & Wayanad & $\begin{array}{l}\text { Muthanga, Noolpuzha } \\
\text { Kanoth } \\
\text { Meppadi }\end{array}$ & $\begin{array}{l}\text { Leelavathy \& } \\
\text { Ganesh } 2000\end{array}$ \\
\hline
\end{tabular}


Table 1 Continued.

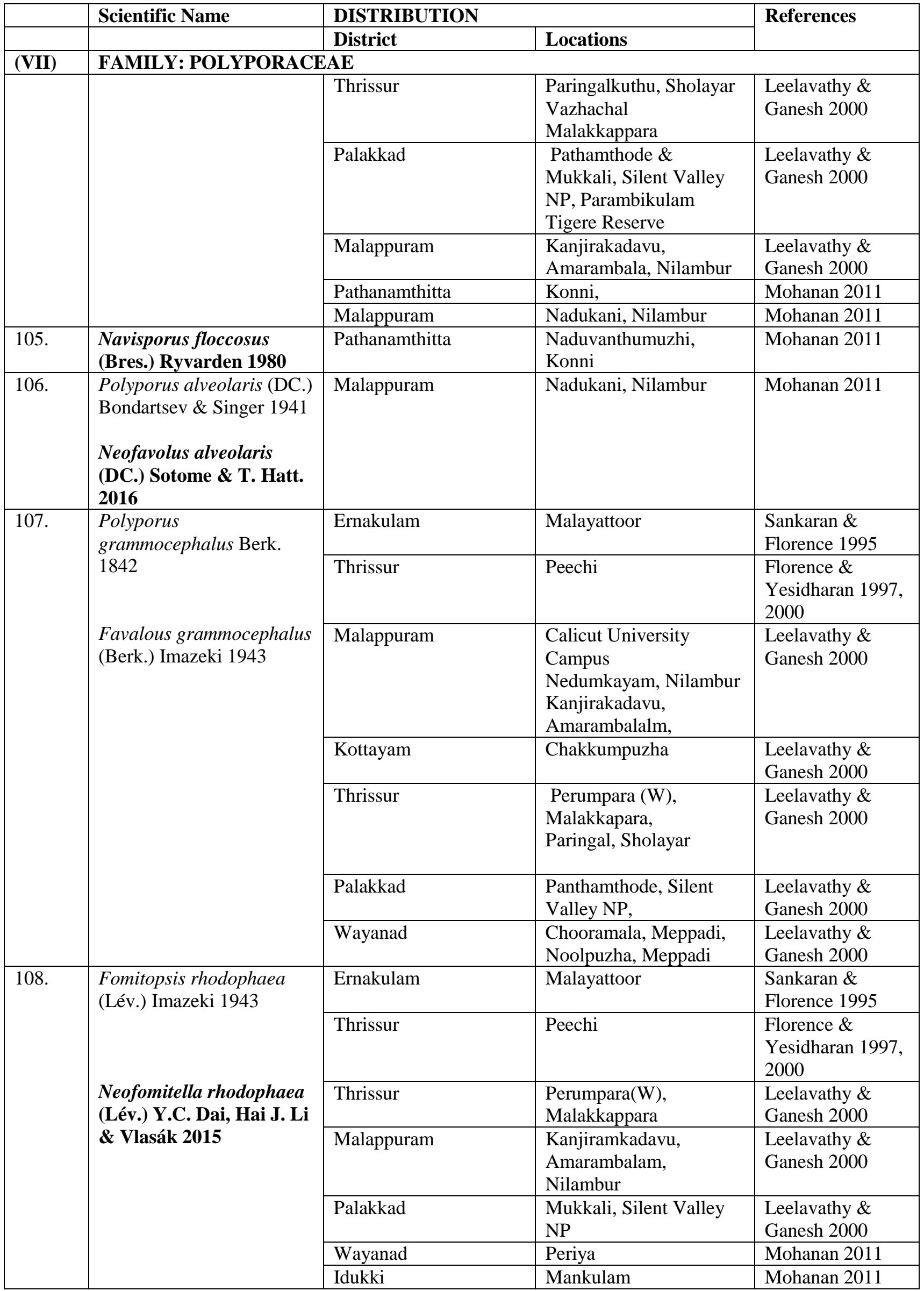


Table 1 Continued.

\begin{tabular}{|c|c|c|c|c|}
\hline & Scientific Name & \multicolumn{2}{|c|}{ DISTRIBUTION } & \multirow[t]{2}{*}{ References } \\
\hline & & District & Locations & \\
\hline (VII) & \multicolumn{4}{|c|}{ FAMILY: POLYPORACEAE } \\
\hline \multirow[t]{3}{*}{109.} & \multirow{3}{*}{$\begin{array}{l}\text { Nigroporus durus } \\
\text { (Jungh.) Murrill } 1907\end{array}$} & Pathanamthitta & Konni & Mohanan 2011 \\
\hline & & Ernakulam & $\begin{array}{l}\text { Iringole Kavu, } \\
\text { Perumbavoor }\end{array}$ & Mohanan 2011 \\
\hline & & Kollam & Shenkily, Kulathupuzha & Mohanan 2011 \\
\hline \multirow[t]{9}{*}{110.} & \multirow[t]{9}{*}{$\begin{array}{l}\text { Nigroporus vinosus } \\
\text { (Berk.) Murrill } 1905\end{array}$} & Thrissur & $\begin{array}{l}\text { Perumpara }(\mathrm{W}), \\
\text { Malakkapara, }\end{array}$ & $\begin{array}{l}\text { Leelavathy \& } \\
\text { Ganesh } 2000\end{array}$ \\
\hline & & Malappuram & Nadigani, Nilambur & $\begin{array}{l}\text { Leelavathy \& } \\
\text { Ganesh } 2000\end{array}$ \\
\hline & & Palakkad & Silent Valley NP & $\begin{array}{l}\text { Leelavathy \& } \\
\text { Ganesh } 2000\end{array}$ \\
\hline & & Wayanad & Mundakai, Meppadi & $\begin{array}{l}\text { Leelavathy \& } \\
\text { Ganesh } 2000\end{array}$ \\
\hline & & Malappuram & $\begin{array}{l}\text { Chandhakkunnu, } \\
\text { Nilambur }\end{array}$ & Mohanan 2011 \\
\hline & & Wayanad & Chembra Hill, Kalpetta & Mohanan 2011 \\
\hline & & Ernakulam & $\begin{array}{l}\text { Iringole Kavu, } \\
\text { Perumbavoor }\end{array}$ & Mohanan 2011 \\
\hline & & Kollam & $\begin{array}{l}\text { Sasthanada, } \\
\text { Kulathupuzha }\end{array}$ & Mohanan 2011 \\
\hline & & Wayanad & Periya & Mohanan 2011 \\
\hline \multirow[t]{2}{*}{111.} & \multirow{2}{*}{$\begin{array}{l}\text { Panus conchatus (Bull.) } \\
\text { Fr. } 1838 \\
\text { Lentinus torulosus (Pers.) } \\
\text { Lloyd } 1913\end{array}$} & Thrissur & Macgad, Peechi & $\begin{array}{l}\text { Forence \& } \\
\text { Yesodharan } 2000\end{array}$ \\
\hline & & Malappuram & Pandikkad & Mohanan 2011 \\
\hline 112. & $\begin{array}{l}\text { Panus hookerianus } \\
\text { (Berk.) T.W. May \& A.E. } \\
\text { Wood } 1995\end{array}$ & Malappuram & - & $\begin{array}{l}\text { Manimohan et al. } \\
2004 \text { (as Lentinus } \\
\text { hookerianus } \\
\text { Berk.). }\end{array}$ \\
\hline 113. & $\begin{array}{l}\text { Panus indicus Sathe \& } \\
\text { J.T. Daniel } 1981\end{array}$ & Malappuram & - & $\begin{array}{l}\text { Sathe \& Daniel } \\
\text { 1980, Florence } \\
\text { 2004 }\end{array}$ \\
\hline \multirow[t]{2}{*}{114.} & \multirow{2}{*}{$\begin{array}{l}\text { Lentinus strigosus Fr. } 1825 \\
\\
\text { Panus neostrigosus } \\
\text { Drechsler-Santos \& } \\
\text { Wartchow } 2012\end{array}$} & & & $\begin{array}{l}\text { Florence \& } \\
\text { Yesodharan } 2000\end{array}$ \\
\hline & & Malappuram & Poonthanam & Mohanan 2011 \\
\hline \multirow[t]{4}{*}{115.} & \multirow{4}{*}{$\begin{array}{l}\text { Panus similis (Berk. \& } \\
\text { Broome) T.W. May \& } \\
\text { A.E. Wood } 1995\end{array}$} & Malappuram & $\begin{array}{l}\text { Chandhakkunnu, } \\
\text { Nilambur }\end{array}$ & Mohanan 2011 \\
\hline & & & Kochupilakkode swamp, & Mohanan 2011 \\
\hline & & Thiruvananthapuram & Arippa & Mohanan 2011 \\
\hline & & $\begin{array}{l}\text { Wayanad, Kannur, } \\
\text { Thiruvananthapuram }\end{array}$ & - & $\begin{array}{l}\text { Manimohan et al. } \\
2004 \text { (as Lentinus } \\
\text { similis Berk. \& } \\
\text { Broome). }\end{array}$ \\
\hline \multirow[t]{4}{*}{116.} & \multirow[t]{4}{*}{$\begin{array}{l}\text { Perenniporia ochroleuca } \\
\text { (Berk.) Ryvarden } 1972\end{array}$} & Malappuram & $\begin{array}{l}\text { Calicut University } \\
\text { Campus }\end{array}$ & $\begin{array}{l}\text { Leelavathy \& } \\
\text { Ganesh } 2000\end{array}$ \\
\hline & & Kannur & Kannur & $\begin{array}{l}\text { Leelavathy \& } \\
\text { Ganesh } 2000\end{array}$ \\
\hline & & Idukki & Mannavanshola, Munnar & Mohanan 2011 \\
\hline & & Thrissur & Sidhan Pocket, Sholayar & Mohanan 2011 \\
\hline
\end{tabular}


Table 1 Continued.

\begin{tabular}{|c|c|c|c|c|}
\hline & Scientific Name & \multicolumn{2}{|c|}{ DISTRIBUTION } & \multirow[t]{2}{*}{ References } \\
\hline & & District & Locations & \\
\hline (VII) & \multicolumn{4}{|c|}{ FAMILY: POLYPORACEAE } \\
\hline 117. & $\begin{array}{l}\text { Polyporus melanopus } \\
\text { (Pers.) Fr. } 1821 \\
\text { Picipes melanopus (Pers.) } \\
\text { Zmitr. \& Kovalenko } \\
\mathbf{2 0 1 6}\end{array}$ & Malappuram & $\begin{array}{l}\text { Chandhakkunnu, } \\
\text { Nilambur }\end{array}$ & Mohanan 2011 \\
\hline 118. & $\begin{array}{l}\text { Polyporus virgatus Berk. } \\
\text { \& M.A. Curtis } 1868 \\
\text { Picipes virgatus (Berk. \& } \\
\text { M.A. Curtis) J.L. Zhou } \\
\text { \& B.K. Cui 2016 }\end{array}$ & Malappuram & $\begin{array}{l}\text { Manacherry, Nilambur } \\
\text { Karulai, Nilambur }\end{array}$ & Mohanan 2011 \\
\hline \multirow[t]{2}{*}{119.} & \multirow[t]{2}{*}{$\begin{array}{l}\text { Piloporia indica Ganesh } \\
\text { \& Ryvarden } 1988\end{array}$} & Palakkad & Silent Valley NP & $\begin{array}{l}\text { Ganesh et al. } \\
1988\end{array}$ \\
\hline & & Malappuram & $\begin{array}{l}\text { Kaduvakunnu, } \\
\text { Nadukani, Nilambur }\end{array}$ & $\begin{array}{l}\text { Leelavathy \& } \\
\text { Ganesh } 2000\end{array}$ \\
\hline \multirow[t]{15}{*}{120.} & \multirow[t]{15}{*}{$\begin{array}{l}\text { Polyporus dictyopus } \\
\text { Mont. } 1835\end{array}$} & Wayanad & $\begin{array}{l}\text { Mundakai, Meppadi, } \\
\text { Chembra peak, Meppadi }\end{array}$ & $\begin{array}{l}\text { Leelavathy \& } \\
\text { Ganesh } 2000\end{array}$ \\
\hline & & Palakkad & \begin{tabular}{|l|} 
Nelliampathy \\
\end{tabular} & Mohanan 2011 \\
\hline & & Thrissur & Kuthiran & Mohanan 2011 \\
\hline & & Malappuram & $\begin{array}{l}\text { Chandhakkunnu, } \\
\text { Nilambur }\end{array}$ & Mohanan 2011 \\
\hline & & Thrissur & $\begin{array}{l}\text { Pukayilappara, } \\
\text { Vazhachal }\end{array}$ & Mohanan 2011 \\
\hline & & Idukki & $\begin{array}{l}\text { Pampadumshola, } \\
\text { Munnar }\end{array}$ & Mohanan 2011 \\
\hline & & Ernakulam & Malayattoor & $\begin{array}{l}\text { Sankaran \& } \\
\text { Florence } 1995\end{array}$ \\
\hline & & Thrissur & Peechi & $\begin{array}{l}\text { Florence \& } \\
\text { Yesodharan, } \\
\text { 1997, 2000 }\end{array}$ \\
\hline & & Thiruvananthapuram & Arippa & Mohanan 2011 \\
\hline & & Thrissur & Kuthiran & Mohanan 2011 \\
\hline & & Malappuram & $\begin{array}{l}\text { Calicut University } \\
\text { Campus } \\
\text { Nedumkayam, Nilambur } \\
\text { Kanjirakadavu, } \\
\text { Amarambalalm, }\end{array}$ & $\begin{array}{l}\text { Leelavathy \& } \\
\text { Ganesh } 2000\end{array}$ \\
\hline & & Kottayam & Chakkumpuzha & $\begin{array}{l}\text { Leelavathy \& } \\
\text { Ganesh } 2000\end{array}$ \\
\hline & & Thrissur & $\begin{array}{l}\text { Perumpara }(\mathrm{W}), \\
\text { Malakkapara } \\
\text { Paringal, Sholayar }\end{array}$ & $\begin{array}{l}\text { Leelavathy \& } \\
\text { Ganesh } 2000\end{array}$ \\
\hline & & Palakkad & $\begin{array}{l}\text { Panthamthode, Silent } \\
\text { Valley NP, }\end{array}$ & $\begin{array}{l}\text { Leelavathy \& } \\
\text { Ganesh } 2000\end{array}$ \\
\hline & & Wayanad & $\begin{array}{l}\text { Chooramala, Meppadi, } \\
\text { Noolpuzha, Meppadi }\end{array}$ & $\begin{array}{l}\text { Leelavathy \& } \\
\text { Ganesh } 2000\end{array}$ \\
\hline \multirow[t]{2}{*}{121.} & \multirow{2}{*}{$\begin{array}{l}\text { Polyporus hemicapnodes } \\
\text { Berk. \& Broome } 1873 \\
\text { Polyporus leprieurii } \\
\text { Mont. 1840 }\end{array}$} & Thrissur & $\begin{array}{l}\text { Perumpara }(\mathrm{W}) \\
\text { Malakkapara, Sholayar }\end{array}$ & $\begin{array}{l}\text { Leelavathy \& } \\
\text { Ganesh } 2000\end{array}$ \\
\hline & & Idukki & Anamudishola, Munnar & Mohanan 2011 \\
\hline
\end{tabular}


Table 1 Continued.

\begin{tabular}{|c|c|c|c|c|}
\hline & Scientific Name & \multicolumn{2}{|c|}{ DISTRIBUTION } & \multirow[t]{2}{*}{ References } \\
\hline & & District & Locations & \\
\hline (VII) & \multicolumn{4}{|c|}{ FAMILY: POLYPORACEAE } \\
\hline 122. & $\begin{array}{l}\text { Poria hypobrunnea Petch } \\
1916\end{array}$ & Kottayam & $\begin{array}{l}\text { Mundakkayam } \\
\text { Chethackal }\end{array}$ & $\begin{array}{l}\text { Rajalekshmy \& } \\
\text { Pillai 1978, } \\
\text { Bilgrami et al. } \\
1991\end{array}$ \\
\hline 123. & $\begin{array}{l}\text { Polyporus rugulosus Lév. } \\
1844\end{array}$ & Idukki & Anamudishola, Munnar & Mohanan 2011 \\
\hline 124. & $\begin{array}{l}\text { Pycnoporus cinnabarinus } \\
\text { (Jacq.) P. Karst. } 1881\end{array}$ & Thrissur & Peechi WLS & Iqbal et al. 2016 \\
\hline \multirow[t]{9}{*}{125.} & \multirow[t]{9}{*}{$\begin{array}{l}\text { Pycnoporus sanguineus } \\
\text { (L.) Murrill } 1904\end{array}$} & Ernakulam & Malayattoor & $\begin{array}{l}\text { Sankaran \& } \\
\text { Florence } 1995\end{array}$ \\
\hline & & Thrissur & Peechi & $\begin{array}{l}\text { Florence \& } \\
\text { Yesodharan } 1997 \\
2000\end{array}$ \\
\hline & & Wayanad & Kuppadi, Sultan Battery & $\begin{array}{l}\text { Leelavathy \& } \\
\text { Ganesh } 2000\end{array}$ \\
\hline & & Malappuram & $\begin{array}{l}\text { Calicut University } \\
\text { Campus }\end{array}$ & $\begin{array}{l}\text { Leelavathy \& } \\
\text { Ganesh } 2000\end{array}$ \\
\hline & & Palakkad & Silent Valley NP & $\begin{array}{l}\text { Leelavathy \& } \\
\text { Ganesh } 2000\end{array}$ \\
\hline & & Thiruvananthapuram & Unnippara, Palode & Mohanan 2011 \\
\hline & & Thiruvananthapuram & Ammayambalam, & Mohanan 2011 \\
\hline & & Kollam & Kulathupuzha & Mohanan 2011 \\
\hline & & Pathanamthitta & Konni & Mohanan 2011 \\
\hline \multirow[t]{4}{*}{126.} & \multirow{4}{*}{$\begin{array}{l}\text { Pyrofomes } \\
\text { albomarginatus (Zipp. ex } \\
\text { Lév.) Ryvarden } 1972\end{array}$} & Ernakulam & \begin{tabular}{|l|} 
Iringole Kavu, \\
Perumbavoor \\
\end{tabular} & Mohanan 2011 \\
\hline & & Thiruvananthapuram & Arippa & Mohanan 2011 \\
\hline & & Malappuram & Karulai, Nilambur & Mohanan 2011 \\
\hline & & Thrissur & $\begin{array}{l}\text { Perumpara (W), } \\
\text { Malakkapara, Sholayar }\end{array}$ & $\begin{array}{l}\text { Leelavathy \& } \\
\text { Ganesh } 2000\end{array}$ \\
\hline \multirow[t]{6}{*}{127.} & \multirow{6}{*}{$\begin{array}{l}\text { Royoporus spatulatus } \\
\text { (Jungh.) A.B. De } 1996\end{array}$} & Thiruvananthapuram & Arippa & Mohanan 2011 \\
\hline & & Malappuram & Karulai, Nilambur & Mohanan 2011 \\
\hline & & Wayanad & Chandanathode & Mohanan 2011 \\
\hline & & Palakkad & Nelliampathy & Mohanan 2011 \\
\hline & & Thiruvananthapuram & Ammayambalam & Mohanan 2011 \\
\hline & & Kolllam & Kulathupuzha & Mohanan 2011 \\
\hline \multirow[t]{3}{*}{128.} & \multirow{3}{*}{$\begin{array}{l}\text { Skeletocutis nivea } \\
\text { (Jungh.) Jean Keller } \\
1979\end{array}$} & Ernakulam & Malayattoor & $\begin{array}{l}\text { Sankaran \& } \\
\text { Florence } 1995\end{array}$ \\
\hline & & Malappuram & $\begin{array}{l}\text { Calicut University } \\
\text { Campus } \\
\text { Kadavakunnu, } \\
\text { Nadugani, Nilambur }\end{array}$ & $\begin{array}{l}\text { Leelavathy \& } \\
\text { Ganesh } 2000\end{array}$ \\
\hline & & Palakkad & $\begin{array}{l}\text { Vengoli peak, } \\
\text { Parambikulam Tgere } \\
\text { Reserve }\end{array}$ & $\begin{array}{l}\text { Leelavathy \& } \\
\text { Ganesh } 2000\end{array}$ \\
\hline \multirow[t]{2}{*}{129.} & $\begin{array}{l}\text { Coriolopsis telfairii } \\
\text { (Klotzsch) Ryvarden } 1972\end{array}$ & Malappuram & $\begin{array}{l}\text { Nilambur } \\
\text { Kaduvakunnu, } \\
\text { Nadugani, Nilambur }\end{array}$ & $\begin{array}{l}\text { Leelavathy \& } \\
\text { Ganesh } 2000\end{array}$ \\
\hline & $\begin{array}{l}\text { Trametella telfairii } \\
\text { (Klotzsch) M. Pieri \& B. } \\
\text { Rivoire 2008 }\end{array}$ & Wayanad & $\begin{array}{l}\text { Karadimala, Brahmagiri, } \\
\text { Thirunelli }\end{array}$ & $\begin{array}{l}\text { Leelavathy \& } \\
\text { Ganesh } 2000\end{array}$ \\
\hline
\end{tabular}


Table 1 Continued.

\begin{tabular}{|c|c|c|c|c|}
\hline & Scientific Name & \multicolumn{2}{|c|}{ DISTRIBUTION } & \multirow[t]{2}{*}{ References } \\
\hline & & District & Locations & \\
\hline (VII) & \multicolumn{4}{|c|}{ FAMILY: POLYPORACEAE } \\
\hline \multirow[t]{2}{*}{130.} & \multirow{2}{*}{$\begin{array}{l}\text { Hexagonia apiaria (Pers.) } \\
\text { Fr. } 1838 \\
\\
\text { Trametes apiaria } \text { (Pers.) } \\
\text { Zmitr., Wasser \& Ezhov } \\
\text { 2012 }\end{array}$} & Thrissur & Peechi & $\begin{array}{l}\text { Florence \& } \\
\text { Yesodharan } 2000\end{array}$ \\
\hline & & Malappuram & Ingar & Mohanan 2011 \\
\hline \multirow[t]{5}{*}{131.} & \multirow[t]{5}{*}{$\begin{array}{l}\text { Trametes cingulata Berk. } \\
1854\end{array}$} & Palakkad & $\begin{array}{l}\text { Vengoli peak, } \\
\text { Tunakadavu, } \\
\text { Parambikulam Tiger } \\
\text { Reserve Pathamthodu, } \\
\text { Silent Valley NP } \\
\end{array}$ & $\begin{array}{l}\text { Leelavathy \& } \\
\text { Ganesh } 2000\end{array}$ \\
\hline & & Thrissur & Vattilappara, Vazhachal & $\begin{array}{l}\text { Leelavathy \& } \\
\text { Ganesh } 2000\end{array}$ \\
\hline & & Malappuram & $\begin{array}{l}\text { Calicut University } \\
\text { Campus }\end{array}$ & $\begin{array}{l}\text { Leelavathy \& } \\
\text { Ganesh } 2000\end{array}$ \\
\hline & & Thiruvananthapuram & Arippa & Mohanan 2011 \\
\hline & & Wayanad & Muthanga & Mohanan 2011 \\
\hline \multirow[t]{2}{*}{132.} & \multirow[t]{2}{*}{$\begin{array}{l}\text { Trametes cotonea (Pat. \& } \\
\text { Har.) Ryvarden } 1972\end{array}$} & Wayanad & $\begin{array}{l}\text { Noolpuzha, Muthanga } \\
\text { Mundakai, Meppadi }\end{array}$ & $\begin{array}{l}\text { Leelavathy \& } \\
\text { Ganesh } 2000\end{array}$ \\
\hline & & Pathanamthitta & Vanvila, Pathanapuram & Mohanan 2011 \\
\hline 133. & $\begin{array}{l}\text { Lenzites elegans (Spreng.) } \\
\text { Pat. } 1900 \\
\text { Trametes elegans } \\
\text { (Spreng.) Fr. } 1838\end{array}$ & Idukki & $\begin{array}{l}\text { Pampadumshola, } \\
\text { Munnar }\end{array}$ & Mohanan 2011 \\
\hline \multirow[t]{4}{*}{134.} & \multirow{2}{*}{$\begin{array}{l}\text { Daedalea flavida Lév. } \\
1844\end{array}$} & Thiruvananthapuram & Unnippara, Palode & Mohanan 2011 \\
\hline & & Ernakulam & $\begin{array}{l}\text { Iringole kavu, } \\
\text { Perumbavoor }\end{array}$ & Mohanan 2011 \\
\hline & \multirow[b]{2}{*}{$\begin{array}{l}\text { Trametes flavida (Lév.) } \\
\text { Zmitr., Wasser \& Ezhov } \\
2012\end{array}$} & Thrissur & Sidhan Pocket, Sholayar & Mohanan 2011 \\
\hline & & Pathanamthitta & Konni & Mohanan 2011 \\
\hline \multirow[t]{4}{*}{135.} & \multirow[t]{4}{*}{$\begin{array}{l}\text { Trametes hirsuta } \\
\text { (Wulfen) Lloyd } 1924\end{array}$} & Malappuram & $\begin{array}{l}\text { Calicut University } \\
\text { Campus } \\
\text { Kanjirakadavu, } \\
\text { Nilambur }\end{array}$ & $\begin{array}{l}\text { Leelavathy \& } \\
\text { Ganesh } 2000\end{array}$ \\
\hline & & Thrissur & Thiruvambady & $\begin{array}{l}\text { Leelavathy \& } \\
\text { Ganesh } 2000\end{array}$ \\
\hline & & Wayanad & $\begin{array}{l}\text { Karadimala, } \\
\text { Brahmagiri, Thirunelli } \\
\text { Mundakai, Meppadi } \\
\text { Kuppadi, Sultan Battery }\end{array}$ & $\begin{array}{l}\text { Leelavathy \& } \\
\text { Ganesh } 2000\end{array}$ \\
\hline & & Palakkad & Silent Valley NP & $\begin{array}{l}\text { Leelavathy \& } \\
\text { Ganesh } 2000\end{array}$ \\
\hline 136. & $\begin{array}{l}\text { Trametes marianna } \\
\text { (Pers.) Ryvarden } 1973\end{array}$ & Wayanad & Mundakai, Meppadi & $\begin{array}{l}\text { Leelavathy \& } \\
\text { Ganesh } 2000\end{array}$ \\
\hline 137. & $\begin{array}{l}\text { Trametes maxima (Mont.) } \\
\text { A. David \& Rajchenb. } \\
1985\end{array}$ & Thiruvananthapuram & Unnippara, Palode & Mohanan 2011 \\
\hline
\end{tabular}


Table 1 Continued.

\begin{tabular}{|c|c|c|c|c|}
\hline & Scientific Name & \multicolumn{2}{|c|}{ DISTRIBUTION } & \multirow[t]{2}{*}{ References } \\
\hline & & District & Locations & \\
\hline (VII) & \multicolumn{4}{|c|}{ FAMILY: POLYPORACEAE } \\
\hline \multirow[t]{3}{*}{138.} & \multirow[t]{3}{*}{$\begin{array}{l}\text { Trametes modesta (Kunze } \\
\text { ex Fr.) Ryvarden } 1972\end{array}$} & Malappuram & $\begin{array}{l}\text { Kaduvakunnu, } \\
\text { Nadugani, Nilambur }\end{array}$ & $\begin{array}{l}\text { Leelavathy \& } \\
\text { Ganesh } 2000\end{array}$ \\
\hline & & Palakkad & Silent Valley NP & $\begin{array}{l}\text { Leelavathy \& } \\
\text { Ganesh } 2000\end{array}$ \\
\hline & & Thiruvananthapuram & Arippa & Mohanan 2011 \\
\hline 139. & $\begin{array}{l}\text { Trametes pubescens } \\
\text { (Schumach.) Pilát } 1939\end{array}$ & Idukki & $\begin{array}{l}\text { Pambadumshola, } \\
\text { Munnar }\end{array}$ & Mohanan 2011 \\
\hline 140. & $\begin{array}{l}\text { Trametes varians Van der } \\
\text { Byl } 1922\end{array}$ & Wayanad & Kuppadi, Sultan Battery & \\
\hline 141. & $\begin{array}{l}\text { Microporus vernicipes } \\
\text { (Berk.) Kuntze } 1898 \\
\text { Trametes vernicipes } \\
\text { (Berk.) Zmitr., Wasser \& } \\
\text { Ezhov } 2012\end{array}$ & Wayanad & Chandanathode & Mohanan 2011 \\
\hline 142. & $\begin{array}{l}\text { Trametes versicolor }(\mathrm{L} .) \\
\text { Lloyd } 1921\end{array}$ & Malappuram & $\begin{array}{l}\text { Chandhanakkunnu, } \\
\text { Nilambur }\end{array}$ & Mohanan 2011 \\
\hline 143. & $\begin{array}{l}\text { Lenzites vespaceus (Pers.) } \\
\text { Pat. } 1900 \\
\text { Trametes vespacea (Pers.) } \\
\text { Zmitr., Wasser \& Ezhov } \\
\mathbf{2 0 1 2}\end{array}$ & Pathanamthitta & Konni & Mohanan 2011 \\
\hline 144. & $\begin{array}{l}\text { Trametes villosa }(\mathrm{Sw} .) \\
\text { Kreisel } 1971\end{array}$ & Malappuram & $\begin{array}{l}\text { Chandhanakkunnu, } \\
\text { Nilambur }\end{array}$ & Mohanan 2011 \\
\hline \multirow[t]{8}{*}{145 . } & \multirow[t]{8}{*}{$\begin{array}{l}\text { Trichaptum biforme (Fr.) } \\
\text { Ryvarden } 1972\end{array}$} & Malappuram & Nilambur & $\begin{array}{l}\text { Leelavathy \& } \\
\text { Ganesh } 2000\end{array}$ \\
\hline & & Palakkad & Silent Valley NP & $\begin{array}{l}\text { Leelavathy \& } \\
\text { Ganesh } 2000\end{array}$ \\
\hline & & Wayanad & Chembra peak, Meppadi & $\begin{array}{l}\text { Leelavathy \& } \\
\text { Ganesh } 2000\end{array}$ \\
\hline & & Idukki & $\begin{array}{l}\text { Mannavanshola, } \\
\text { Munnar, }\end{array}$ & Mohanan 2011 \\
\hline & & Idukki & $\begin{array}{l}\text { Pambadumshola, } \\
\text { Munnar }\end{array}$ & Mohanan 2011 \\
\hline & & Idukki & Anamudishola, Munnar & Mohanan 2011 \\
\hline & & Idukki & Devikulam, Munnar & Mohanan 2011 \\
\hline & & Idukki & Kundala, Munnar & Mohanan 2011 \\
\hline 146. & $\begin{array}{l}\text { Trichaptum byssogenum } \\
\text { (Jungh.) Ryvarden } 1972\end{array}$ & Palakkad & $\begin{array}{l}\text { Kamathalamudi, } \\
\text { Tunakadavu, } \\
\text { Parambikulam Tiger } \\
\text { Reserve } \\
\end{array}$ & $\begin{array}{l}\text { Leelavathy \& } \\
\text { Ganesh } 2000\end{array}$ \\
\hline (VIII) & \multicolumn{4}{|c|}{ FAMILY: SCHIZOPORACEAE } \\
\hline 147. & $\begin{array}{l}\text { Oxyporus mollissimus } \\
\text { (Pat.) D.A. Reid } 1975 \\
\text { Leucophellinus hobsonii } \\
\text { (Berk. ex Cooke) } \\
\text { Ryvarden 1988 }\end{array}$ & Palakkad & $\begin{array}{l}\text { Kamathalamudi, } \\
\text { Tunakadavu, } \\
\text { Parambikulam Tiger } \\
\text { Reserve }\end{array}$ & $\begin{array}{l}\text { Leelavathy \& } \\
\text { Ganesh } 2000\end{array}$ \\
\hline \multirow[t]{3}{*}{148.} & \multirow[t]{3}{*}{$\begin{array}{l}\text { Schizopora paradoxa } \\
\text { (Schrad.) Donk } 1967\end{array}$} & Idukki & $\begin{array}{l}\text { Pambadumshola, } \\
\text { Munnar }\end{array}$ & Mohanan 2011 \\
\hline & & Idukki & Anamudishola, Munnar & Mohanan 2011 \\
\hline & & Idukki & Mannavanshola, Munnar & Mohanan 2011 \\
\hline
\end{tabular}




\section{References}

Bagchee K. 1954 - The fungal disease of Sal (Shorea robusta Gaertn. f. \& Fils.), Part II, secondary parasites of Sal. Indian For. Rec. 8, 97-184.

Bilgrami KS, Jamaludhdin S, Rizwi MA. 1991 - Fungi of India. List and References. Today and Tommorrow's Printers and Publishers, New Delhi. 798p.

Farook VA, Khan SS, Manimohan P. 2013 - A checklist of agarics (gilled mushrooms) of Kerala State, India. Mycosphere 4(1), 97-131.

Florence EJM, Balasundaran M. 2000 - Mushroom cultivation using forest litter and waste wood. KFRI Research Report No. 195. Kerala Forest Research Institute, Peechi, Kerala, India.

Florence EJM, Yesodharan K. 1997 - Status survey of macrofungal flora of Peechi-Vazhani wildlife sanctuary. In: Proceedings of Ninth Kerala Science Congress (ed PK Iyengar). STEC, Thiruvananthauram, 477-479.

Florence EJM, Yesodharan K. 2000 - macrofungal flora of Peechi-Vazhani wildlife sanctuary. KFRI Research report No. 191.

Florence EJM. 2004 - Biodiversity Documentation for Kerala, Part 2: Microorganisms (Fungi). KFRI Handbook No.17. Kerala Forest Research Institute, Peechi, Kerala, India.pp 292.

Ganesh PN, Leelavath KM. 1986 - New Records of Phellinus from India. Current Science. 55 (15), 726-728.

Ganesh PN, Leelavath KM, Ryvarden L. 1988 - Piloporia indica sp. nov. from India. Transactions of the British Mycological Society 9(1), 169-171.

Geetha D, Nair MC, Bhavanidevi S. 1989 - Mushroom mycelia as poultry feed. Indian Phytopathology 42(3), 462-463.

Geetha D, Sherin AS, Gokulapalan C, Suhrban M. 2002 - Pleurotus tuber-regium - a new promising mushroom for the tropics. Mushroom Research 11(2), 73-76.

Hosagoudar VB. 1996 - Meliolates of India, Botanical survey of India, Culcutta. 363p.

Iqbal AM, Vidyasagaran K, Ganesh PN. 2016 - New records of polypores (Basidiomycota: Aphyllophorales) from the southern Western Ghats with an identification key for polypores in Peechi-Vazhani Wildlife Sanctuary,Kerala, India. Journal of Threatened Taxa. 8(9),91989207.

Joseph AV, Abraham TK, Vrinda KB, Pradeep CK. 1995 - New agarics from South India. Mushroom Research 4, 1-6.

Kumar TKA, Manimohan P. 2005 - A new species of Lentinus from India. Mycotaxon 92, 119-123.

Leelavathy KM, Ganesh PN. 2000 - Polypores of Kerala. Daya Publishing House, Delhi, pp 165.

Manimohan P, Divya N, Kumar TKA, Vrinda KB, Pradeep CK - 2004. The genus Lentinus in Kerala State, India. Mycotaxon 90(2), 311-318.

Manimohan P, Leelavathy KM. 1995 - A new variety of Lentinus caespiticola from Southern India. Mycological Research 99(4), 451-452.

Mohanan C. 2011 - Macrofungi of Kerala. KFRI Handbook No. 27, Kerala Forest Research Institute, Peechi, Kerala, India.

Mohanan C. 1994 - Decay of standing trees in natural forests. KFRI Handbook No. 97. Kerala Forest Research Institute, Peechi, Kerala, India, pp 34.

Ouseph A, Geetha D, Suharban M. 2001 - Lignocellulose degradation by oyster mushroom. Mushroom Research 10(1), 37-40.

Pradeep CK, Vrinda KB. 2007 - Some noteworthy agarics from Western Ghats of Kerala. Journal of Mycopathological Research (1), 1-14.

Rajalekshmy VK, Radhakrishnan PPN. 1978 - Poria root rot of rubbering India. Indian phytopath. 31,199-202.

Rangaswami G, Seshadn VS, Lucy Channamma KA. 1970 - Fungi of South India. University of Agricultural. Sciences, Bangalore, pp 193.

Reddy CS, Pattanaik C, Reddy KN, Raju VS. 2007 - Census of endemic flowering plants of Kerala, India. Journal of Plant Sciences, 2, 489-503. 
Sankaran KV, Florence EJM. 1995 - Macrofungal flora and checklist of plant diseases of Malayattoor forests (Kerala). Advances in Forestry Research in India. (ed SK Mukherjee) International Book Distributors, Dehra Dun, 12, 147-168.

Sasidharan N. 2004 - Forest Trees of Kerala. KFRI. Peechi. 166 p.

Sathe AV, Daniel J. 1980 - Agaricales (Mushrooms) of Kerala State. MACS Monograph. No. 1, 75108.

Sharma JK, Mohanan C, Florence EJM. 1985 - Disease survey in nurseries and plantations of forest tree species grown in Kerala. KFRI Research Report No. 36. Kerala Forest Research Institute, Peechi, Kerala, India.

Varghese SP, Pradeep CK, Vrida KB. 2010 - Mushrooms of tribal importance in Wayanad area of Kerala. Journal of Mycopathological Research 48(2), 311-320. 